
COMMUNICATIONS

ISSN 2056-9890

Received 30 September 202

Accepted 7 October 2021

Edited by L. Van Meervelt, Katholieke Universiteit Leuven, Belgium

Keywords: crystal structure; UCH-L1 activator; alanylpiperidine derivatives; polymorph risk assessment.

CCDC references: 2114340; 2114339

Supporting information: this article has supporting information at journals.iucr.org/e

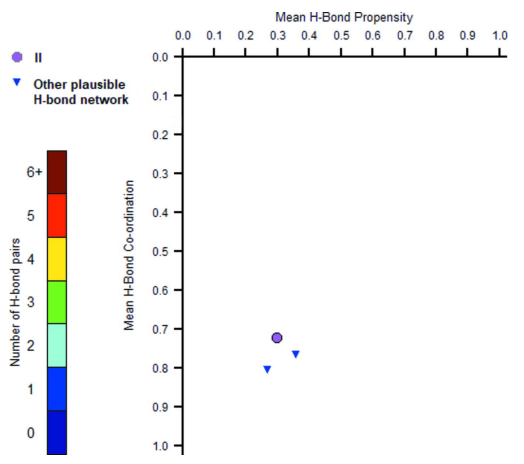

OPEN Ә ACCESS

\section{Crystal structures of two alanylpiperidine analogues}

\author{
Kalina Mambourg, ${ }^{a} *$ Nikolay Tumanov, ${ }^{a}$ Gilles Henon, ${ }^{a}$ Steve Lanners, ${ }^{a}$ Javier \\ Garcia-Ladona $^{\mathbf{b}}$ and Johan Wouters ${ }^{\mathrm{a}}$
}

aDepartment of Chemistry, University of Namur, Rue de Bruxelles 61, Namur 5000, Belgium, and ${ }^{\mathbf{b}}$ Abaxys Therapeutics,
Rue du Berceau 91, Villers-la-Ville 1495, Belgium. *Correspondence e-mail: kalina.mambourg@unamur.be

The structure of ethyl 1-[N-(4-methylphenyl)- $N$-(methylsulfonyl)alanyl]piperidine-4-carboxylate, $\mathrm{C}_{19} \mathrm{H}_{28} \mathrm{~N}_{2} \mathrm{O}_{5} \mathrm{~S}$, I, a compound of interest as activator of Ubiquitin C-terminal Hydrolase-L1 (UCH-L1), was determined by singlecrystal X-ray diffraction (SCXRD) analysis. In order to find new activators, a derivative of compound I, namely, 1-[ $N$-(4-methylphenyl)- $N$-(methylsulfonyl)alanyl]piperidine-4-carboxylic acid, $\mathrm{C}_{17} \mathrm{H}_{24} \mathrm{~N}_{2} \mathrm{O}_{5} \mathrm{~S}$, II, was studied. The synthesis and crystal structure are also reported. Despite being analogues, different crystal packings are observed. Compound II bears a carboxylic group, which favors a strong hydrogen bond. A polymorph risk assessment was carried out to study interactions in compound $\mathbf{I I}$.

\section{Chemical context}

Ubiquitin C-terminal Hydrolase-L1 is a deubiquitinase that represents $2 \%$ of the neuronal soluble proteins in the brain and is involved in the neuropathogenesis of neurodegenerative diseases. Studies have shown that several mutations have an impact on the hydrolase activity of UCH-L1 (Leroy et al., 1998; Maraganore et al., 1999) and that its down-regulation is associated with idiopathic Parkinson's disease (Choi et al., 2004). Finding potentiators of UCH-L1 could be a therapeutic pathway for these diseases (Mitsui et al., 2010). Ethyl 1-[N(methylsulfonyl)- $N$-( $p$-tolyl)-alanyl]piperidine-4-carboxylate was discovered through in silico drug screening as an activator of UCH-L1, with a hydrolase activity up to $111 \%$ at $63 \mu \mathrm{M}$ (Mitsui et al., 2010). We studied the only known activator in the literature, compound I. Derivatives of compound I were then investigated as potential activators and compound II was obtained after a saponification. Compound II bears a carboxylic acid group, which opens up the possibility for co-crystallization and salification in order to modulate the physicochemical properties, such as the solubility. We report the crystal structures of these two compounds as well as a survey of the interactions observed in compound $\mathbf{I I}$.<smiles>CCOC(=O)C1CCN(C(=O)C(C)N(c2ccc(C)cc2)S(C)(=O)=O)CC1</smiles>

\section{Structural commentary}

Both compounds crystallize as colorless plate-like crystals but in different space groups. Compound I crystallizes in the 


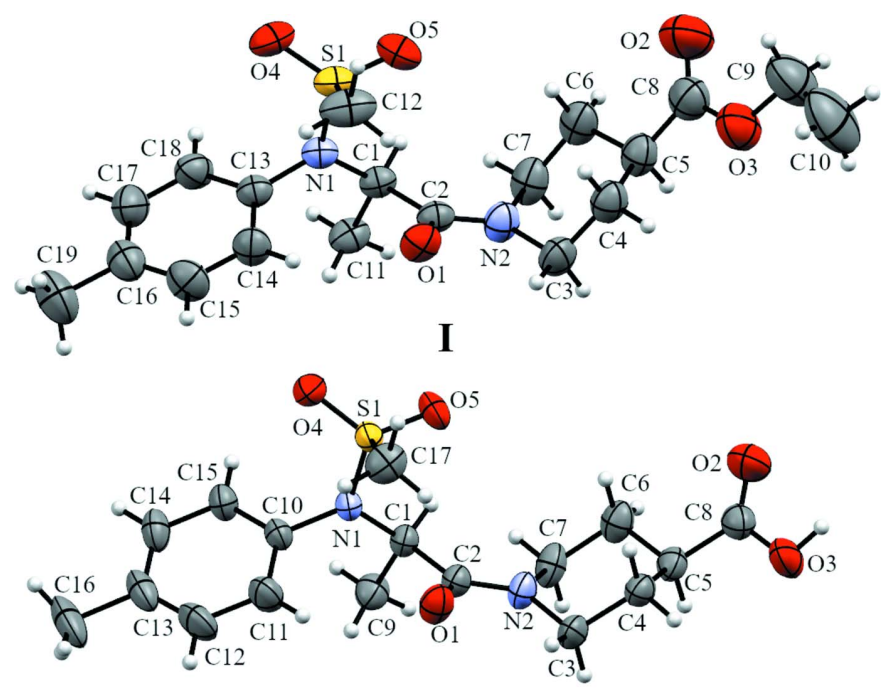

II

Figure 1

The asymmetric units of compounds I and II, with displacement ellipsoids drawn at the $50 \%$ probability level.

triclinic $P \overline{1}$ space groups and compound $\mathbf{I I}$ in the monoclinic $P 2_{1} / n$ space group. The asymmetric units are shown in Fig. 1. Both compounds crystallize as a racemic mixture and have one molecule in the asymmetric unit in a similar conformation. The torsion angle $\mathrm{N} 1-\mathrm{C} 1-\mathrm{C} 2-\mathrm{N} 2$ is $156.2(1)$ and $-153.5(1)^{\circ}$ for $\mathbf{I}$ and $\mathbf{I I}$ respectively. The only slight difference between the two compounds is the geometry of N2. In compound $\mathbf{I}$, the distance between $\mathrm{N} 2$ and the plane formed by C2, C3 and C7 is 0.114 (2) $\AA$ whereas in compound II this distance is $0.014(2) \AA$. A more planar arrangement of $\mathrm{N} 2$ in
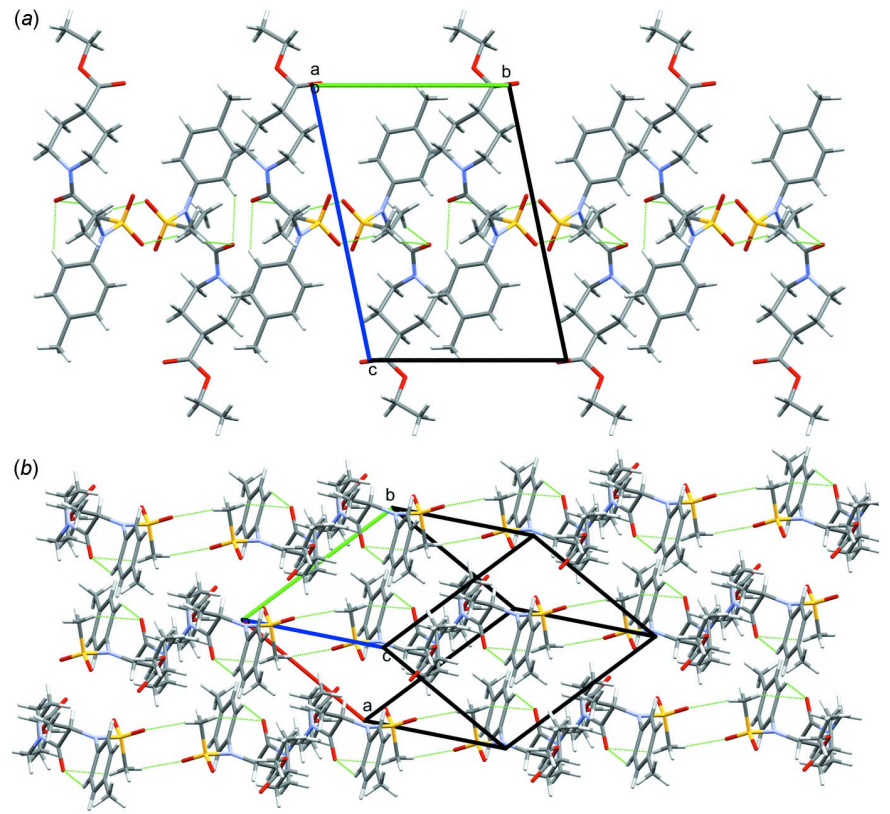

Figure 2

Crystal packing of $\mathbf{I}$ with hydrogen bonds highlighted in green $(a)$ showing one layer of molecules, viewed down the $a$ axis and $(b)$ showing adjacent layers of molecules.
Table 1

Hydrogen-bond geometry $\left(\AA,^{\circ}\right)$ for compound $\mathbf{I}$.

\begin{tabular}{lllll}
\hline$D-\mathrm{H} \cdots A$ & $D-\mathrm{H}$ & $\mathrm{H} \cdots A$ & $D \cdots A$ & $D-\mathrm{H} \cdots A$ \\
\hline $\mathrm{C} 12-\mathrm{H} 12 B \cdots \mathrm{O} 1$ & 0.96 & 2.50 & $3.210(2)$ & 130 \\
$\mathrm{C} 12-\mathrm{H} 12 C \cdots \mathrm{O} 4^{\mathrm{i}}$ & 0.96 & 2.44 & $3.376(2)$ & 164 \\
$\mathrm{C} 14-\mathrm{H} 14 \cdots \mathrm{O} 1$ & 0.93 & 2.48 & $3.177(2)$ & 132 \\
\hline
\end{tabular}

Symmetry code: (i) $-x,-y,-z+1$.

Table 2

Hydrogen-bond geometry $\left(\AA,^{\circ}\right)$ for compound $\mathbf{I I}$.

\begin{tabular}{lllll}
\hline$D-\mathrm{H} \cdots A$ & $D-\mathrm{H}$ & $\mathrm{H} \cdots A$ & $D \cdots A$ & $D-\mathrm{H} \cdots A$ \\
\hline $\mathrm{O} 3-\mathrm{H} 3 \cdots \mathrm{O} 5^{\mathrm{i}}$ & $0.90(3)$ & $1.88(3)$ & $2.7463(15)$ & $161(2)$ \\
$\mathrm{C} 17-\mathrm{H} 17 A \cdots \mathrm{O} 1$ & 0.96 & 2.48 & $3.144(2)$ & 127 \\
$\mathrm{C} 4-\mathrm{H} 4 B \cdots \mathrm{O} 2^{\mathrm{i}}$ & 0.97 & 2.52 & $3.471(2)$ & 167 \\
$\mathrm{C} 11-\mathrm{H} 11 \cdots \mathrm{O} 1$ & 0.93 & 2.56 & $3.2558(19)$ & 132 \\
\hline
\end{tabular}

Symmetry code: (i) $-x+2,-y+1,-z+1$.

compound II is noticed, probably caused by the crystal packing. Single crystals represent the bulk samples as the powder patterns calculated from SCXRD data are similar to the experimental ones.

\section{Supramolecular features}

As compound I does not have any strong hydrogen-bond acceptors, only weak hydrogen bonds are observed in the crystal structure (see Table 1). The amide oxygen atom O1 participates in the formation of two intramolecular hydrogen bonds [ $S_{1}^{1}(7)$ motifs; Etter et al., 1990]. The oxygen atom O4 is inter-connected with atom $\mathrm{H} 12 \mathrm{C}$ of the sulfonyl methyl of an adjacent molecule $[d(\mathrm{H} \cdots \mathrm{O}) 2.44 \AA$; Table 1], forming an $R_{2}^{2}(8)$ hydrogen bond motif along the $a$-axis direction (Fig. 2). As compound $\mathbf{I}$ bears a tolyl moiety, $\pi-\pi$ interactions were expected but were not observed in this crystal packing.

Compound II bearing a carboxylic moiety instead of an ester has an impact on the hydrogen bonds and thus on the

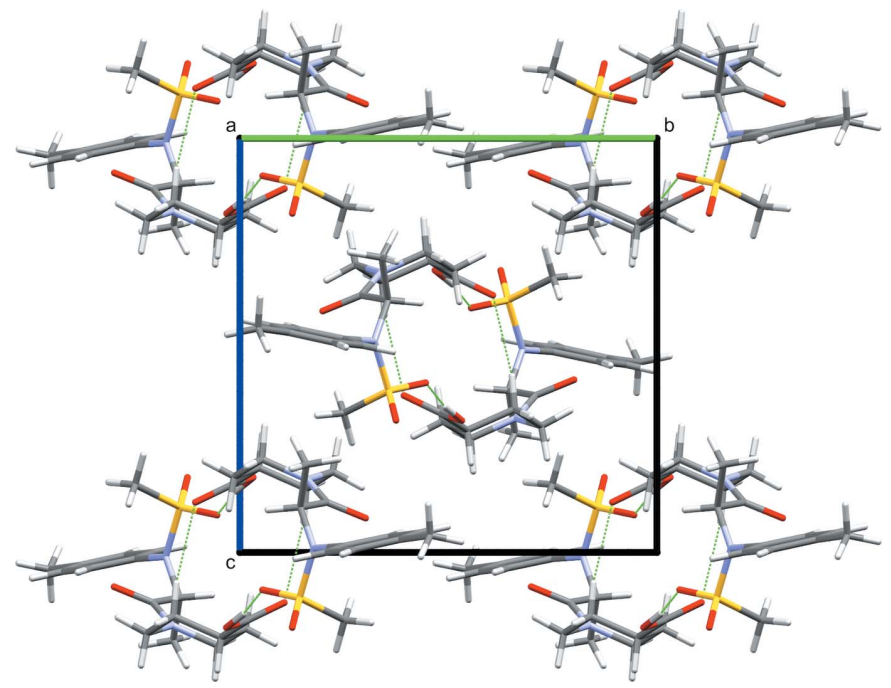

Figure 3

Crystal packing of II showing the tubular arrangement viewed down the $a$ axis. Hydrogen bonds are highlighted in green. 
Table 3

Hydrogen-bond propensity calculation for compound II.

\begin{tabular}{lll}
\hline Donor & Acceptor & Propensity \\
\hline O3 & O2 & 0.36 \\
O3 & O4 & 0.30 \\
O3 & O5 & 0.30 \\
\hline
\end{tabular}

crystal packing. In compound II, a tubular arrangement (Fig. 3) can be observed, which is different from that of compound $\mathbf{I}$. In compound II, a hydrogen-bonded ring with an $R_{2}^{2}(24)$ motif is formed by a strong hydrogen bond between $\mathrm{H} 3$ of the carboxylic acid group and $\mathrm{O} 5$ from an adjacent molecule $[d(\mathrm{H} \cdots \mathrm{O}) 1.88(3) \AA$; Table 2]. In addition, two intramolecular $\left[S_{1}^{1}(7)\right.$ motifs $]$ and one intermolecular $\left[R_{2}^{2}(10)\right.$ motif] weak hydrogen bonds are detected. As in compound $\mathbf{I}$, no $\pi-\pi$ interactions are noticed in the crystal structure. A dimer synthon is observed in the crystal packing in both cases, but for compound $\mathbf{I}$ it is ensured by weak hydrogen bonds in contrast to compound II where the dimer is based on strong hydrogen bonds.

\section{Database survey}

Searches of the Cambridge Structural Database (CSD, version 5.42, update September 2021; Groom et al. 2016) were carried out with the exact structures of compounds I and II and with substructures containing the significant fragments (alanylpiperidine with and without the sulfonyl methyl and tolyl group). No comparable structures came out of this survey.

A polymorph risk assessment based on the hydrogen bonds in the CSD was carried out. This statistical analysis allows us to estimate which atoms are the donors and the acceptors for hydrogen bonds in the crystal structure (Chemburkar et al., 2000; Galek et al., 2007). This quantifies the probability of hydrogen-bond formation and thus the different probable polymorphs that can arise from a specific compound. The results are summarized in Table 3. A hydrogen-bonding interaction between two carboxylic groups is predicted with the highest probability. We did not observe the carboxylic dimer but rather this group interacting with one oxygen of the sulfonyl methyl. The analysis also predicts other plausible hydrogen-bonded networks (Fig. 4), one that is statistically slightly more likely to be formed than the current one. This suggests that another potential polymorph could be obtained. Thus, we undertook a polymorph screening by several crystallization experiments of compound II. The recrystallization solvents that we tested were cyclohexane, toluene, ethyl acetate, chloroform, dichloromethane, acetone, acetonitrile, 2propanol, ethanol and methanol. They all lead to the same polymorph.

\section{Synthesis and crystallization}

Compound I: This was purchased from Evotech (Hamburg, Germany). The product was crystallized by slow evaporation

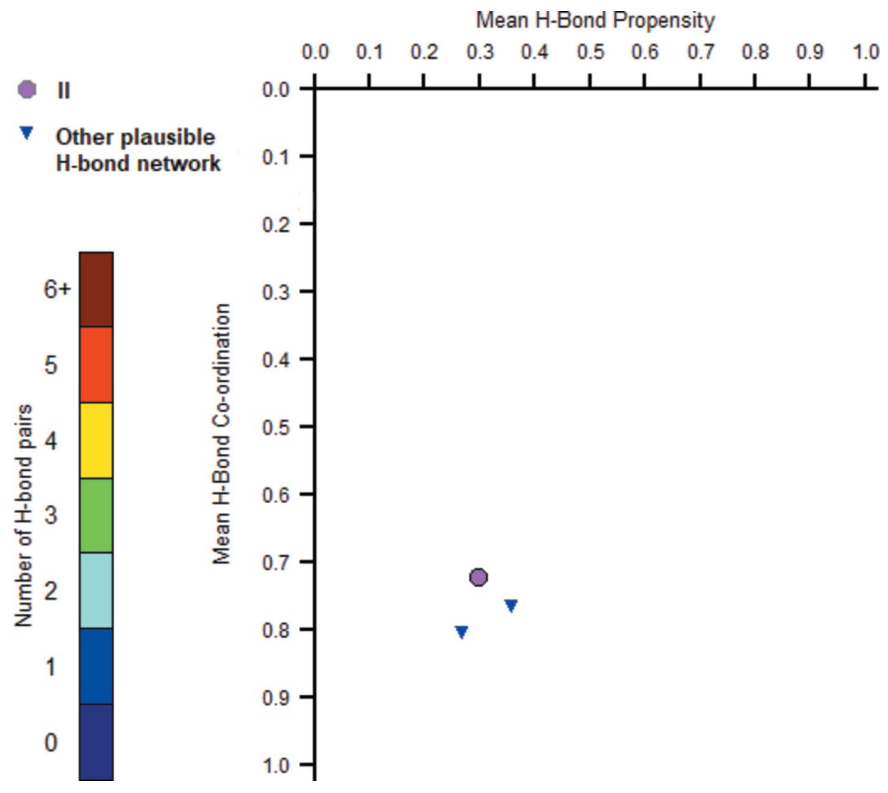

Figure 4

Hydrogen-bond propensity chart for compound II.

from non-anhydrous ethyl acetate, which provided colorless plate-like crystals suitable for SCXRD. M.p. 442.2 K

Compound II: In a round-bottom flask, compound I (405.1 mg, $1.02 \mathrm{mmol}, 1.0 \mathrm{eq}$ ) dissolved in $8 \mathrm{~mL}$ of THF was added to a solution of $\mathrm{LiOH}(81.9 \mathrm{mg}, 3.40 \mathrm{mmol}, 3.4 \mathrm{eq})$ dissolved in $5 \mathrm{~mL}$ of water. The mixture was stirred at room temperature for $8 \mathrm{~h}$. The resulting mixture was washed with ether. The aqueous phase was then acidified with $\mathrm{HCl} 37 \%$ to a $\mathrm{pH}$ of 2 and extracted with dichloromethane. The combined organic phases were dried over anhydrous $\mathrm{Na}_{2} \mathrm{SO}_{4}$ and concentrated under vacuum to yield a white solid $(351.0 \mathrm{mg}$, $93 \%$ ). The product was crystallized by slow evaporation from methanol, which provided colorless plate-like crystals suitable for SCXRD. ${ }^{1} \mathrm{H}$ NMR (DMSO): 12.32 ( $s, 1 \mathrm{H}$, carboxylic acid), $7.39\left(d, 2 \mathrm{H}, \mathrm{CH}_{\text {arom }}\right), 7.20\left(d, 2 \mathrm{H}, \mathrm{CH}_{\text {arom }}\right), 5.20\left(s, 1 \mathrm{H}, \mathrm{CH}_{\alpha}\right)$, 4.03-3.15 (m, 4H, $\left.\mathrm{CH}_{\text {pip }}\right), 2.96\left(s, 3 \mathrm{H}, \mathrm{CH}_{\mathrm{SO} 2 \mathrm{Me}}\right), 2.79(m, 1 \mathrm{H}$, $\left.\mathrm{CH}_{\text {pip }}\right), 2.31$ ( $\left.s, 3 \mathrm{H}, \mathrm{CH}_{\text {PheMe }}\right), 1.83-1.36\left(m, 4 \mathrm{H}, \mathrm{CH}_{\text {pip }}\right), 1.03$ $\left(d, 3 \mathrm{H}, \mathrm{CH}_{\alpha \mathrm{Me}}\right){ }^{13} \mathrm{C} \mathrm{NMR}$ (DMSO): 169.1, 168.7, 138.2, 133.5, 132.0, 129.4, 53.3, 44.5, 41.3, 28.5, 20.7, 16.8. M.p. $496.2 \mathrm{~K}$

\section{Refinement}

Crystal data, data collection and structure refinement details are summarized in Table 4. All $\mathrm{H}$ atoms, except one of the $\mathrm{OH}$ group in II, were refined using a riding model, with $\mathrm{C}-\mathrm{H}$ $=0.93$ (aromatic), 0.96 (methyl) or $0.98 \AA$ (tertiary carbon). Coordinates of the hydrogen atom of the $-\mathrm{OH}$ group were refined. The isotropic atomic displacement parameters of the $\mathrm{H}$ atoms were set at $1.5 U_{\text {eq }}$ of the parent atom for the methyl and alcohol groups, and at $1.2 U_{\mathrm{eq}}$ otherwise.

\section{Acknowledgements}

This work was performed on XRD equipment from the PC2 platform at UNamur and PXRD equipment has been funded 
Table 4

Experimental details.

I

Crystal data

Chemical formula

$M_{\mathrm{r}}$

Crystal system, space group

Temperature $(\mathrm{K})$

$a, b, c(\AA)$

$\alpha, \beta, \gamma\left(^{\circ}\right)$

$V\left(\AA^{3}\right)$

Z

Radiation type

$\mu\left(\mathrm{mm}^{-1}\right)$

Crystal size (mm)

Data collection

Diffractometer

Absorption correction

$T_{\text {min }}, T_{\text {max }}$

No. of measured, independent and observed $[I>2 \sigma(I)]$ reflections

$R_{\text {int }}$

$(\sin \theta / \lambda)_{\max }\left(\AA^{-1}\right)$

Refinement

$R\left[F^{2}>2 \sigma\left(F^{2}\right)\right], w R\left(F^{2}\right), S$

No. of reflections

No. of parameters

$\mathrm{H}$-atom treatment

$\Delta \rho_{\max }, \Delta \rho_{\min }\left(\mathrm{e} \AA^{-3}\right)$

\section{$\mathrm{C}_{19} \mathrm{H}_{28} \mathrm{~N}_{2} \mathrm{O}_{5} \mathrm{~S}$}

396.49

Triclinic, $P \overline{1}$

295

8.5368 (6), 9.6594 (6), 13.5173 (12)

75.947 (6), 79.302 (6), 74.554 (5)

1033.47 (14)

2

Mo $K \alpha$

0.19

$0.79 \times 0.18 \times 0.05$

Oxford Diffraction Xcalibur, Gemini Ultra R

Analytical [CrysAlis PRO (Rigaku OD, 2018),

based on expressions derived by Clark \& Reid (1995)]

$0.923,0.991$

$13200,6870,4304$

0.026

0.762

$0.054,0.158,1.02$

6870

248

$\mathrm{H}$-atom parameters constrained

$0.34,-0.38$
II

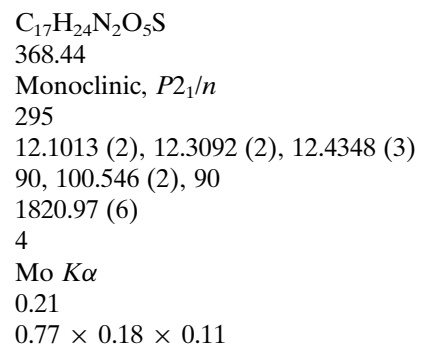

Oxford Diffraction Xcalibur, Gemini Ultra R Analytical [CrysAlis PRO (Rigaku OD, 2018), based on expressions derived by Clark \& Reid (1995)]

$0.882,0.980$

$29518,6284,4779$

0.026

0.761

$0.043,0.126,1.02$

6284

232

$\mathrm{H}$ atoms treated by a mixture of independent and constrained refinement $0.29,-0.29$

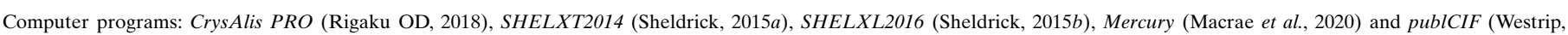
2010).

by FRS-FNRS. The authors thank Laurie Bodard for her help on the polymorph risk assessment.

\section{References}

Chemburkar, S. R., Bauer, J., Deming, K., Spiwek, H., Patel, K., Morris, J., Henry, R., Spanton, S., Dziki, W., Porter, W., Quick, J., Bauer, P., Donaubauer, J., Narayanan, B. A., Soldani, M., Riley, D. \& McFarland, K. (2000). Org. Process Res. Dev. 4, 413-417.

Choi, J., Levey, A. I., Weintraub, S. T., Rees, H. D., Gearing, M., Chin, L. S. \& Li, L. (2004). J. Biol. Chem. 279, 13256-13264.

Clark, R. C. \& Reid, J. S. (1995). Acta Cryst. A51, 887-897.

Etter, M. C., MacDonald, J. C. \& Bernstein, J. (1990). Acta Cryst. B46, 256-262.

Galek, P. T. A., Fábián, L., Motherwell, W. D. S., Allen, F. H. \& Feeder, N. (2007). Acta Cryst. B63, 768-782.

Groom, C. R., Bruno, I. J., Lightfoot, M. P. \& Ward, S. C. (2016). Acta Cryst. B72, 171-179.
Leroy, E., Boyer, R., Auburger, G., Leube, B., Ulm, G., Mezey, E., Harta, G., Brownstein, M. J., Jonnalagada, S., Chernova, T., Dehejia, A., Lavedan, S., Gasser, T., Steinbach, P. J., Wilkinson, K. D. \& Polymeropoulos, M. H. (1998). Nature, 395, 451-452.

Macrae, C. F., Sovago, I., Cottrell, S. J., Galek, P. T. A., McCabe, P., Pidcock, E., Platings, M., Shields, G. P., Stevens, J. S., Towler, M. \& Wood, P. A. (2020). J. Appl. Cryst. 53, 226-235.

Maraganore, D. M., Farrer, M. J., Hardy, J. A., Lincoln, S. J., McDonnell, S. K. \& Rocca, W. A. (1999). Neurology, 53, 18581858.

Mitsui, T., Hirayama, K., Aoki, S., Nishikawa, K., Uchida, K., Matsumoto, T., Kabuta, T. \& Wada, K. (2010). Neurochem. Int. 56, 679-686.

Rigaku OD (2018). CrysAlis PRO. Rigaku Oxford Diffraction, Yarnton, England.

Sheldrick, G. M. (2015a). Acta Cryst. A71, 3-8.

Sheldrick, G. M. (2015b). Acta Cryst. C71, 3-8.

Westrip, S. P. (2010). J. Appl. Cryst. 43, 920-925. 


\section{supporting information}

Acta Cryst. (2021). E77, 1095-1098 [https://doi.org/10.1107/S2056989021010392]

\section{Crystal structures of two alanylpiperidine analogues}

\section{Kalina Mambourg, Nikolay Tumanov, Gilles Henon, Steve Lanners, Javier Garcia-Ladona and Johan Wouters}

\section{Computing details}

For both structures, data collection: CrysAlis PRO (Rigaku OD, 2018); cell refinement: CrysAlis PRO (Rigaku OD, 2018); data reduction: CrysAlis PRO (Rigaku OD, 2018); program(s) used to solve structure: SHELXT2014 (Sheldrick, 2015a); program(s) used to refine structure: SHELXL2016 (Sheldrick, 2015b); molecular graphics: Mercury (Macrae et al., 2020); software used to prepare material for publication: publCIF (Westrip, 2010).

Ethyl 1-[N-(4-methylphenyl)-N-(methylsulfonyl)alanyl]piperidine-4-carboxylate (I)

\section{Crystal data}

$\mathrm{C}_{19} \mathrm{H}_{28} \mathrm{~N}_{2} \mathrm{O}_{5} \mathrm{~S}$

$M_{r}=396.49$

Triclinic, $P \overline{1}$

$a=8.5368(6) \AA$

$b=9.6594(6) \AA$

$c=13.5173(12) \AA$

$\alpha=75.947(6)^{\circ}$

$\beta=79.302(6)^{\circ}$

$\gamma=74.554(5)^{\circ}$

$V=1033.47(14) \AA^{3}$

\section{Data collection}

Oxford Diffraction Xcalibur, Gemini Ultra R diffractometer

Radiation source: fine-focus sealed X-ray tube Graphite monochromator

Detector resolution: 10.3712 pixels $\mathrm{mm}^{-1}$

$\omega$ scans

Absorption correction: analytical

[CrysAlisPro (Rigaku OD, 2018), based on

expressions derived by Clark \& Reid (1995)]

\section{Refinement}

Refinement on $F^{2}$

Least-squares matrix: full

$R\left[F^{2}>2 \sigma\left(F^{2}\right)\right]=0.054$

$w R\left(F^{2}\right)=0.158$

$S=1.02$

6870 reflections

248 parameters

0 restraints

Primary atom site location: dual
$Z=2$

$F(000)=424$

$D_{\mathrm{x}}=1.274 \mathrm{Mg} \mathrm{m}^{-3}$

Mo $K \alpha$ radiation, $\lambda=0.71073 \AA$

Cell parameters from 3246 reflections

$\theta=2.4-30.5^{\circ}$

$\mu=0.19 \mathrm{~mm}^{-1}$

$T=295 \mathrm{~K}$

Plate, colorless

$0.79 \times 0.18 \times 0.05 \mathrm{~mm}$

$T_{\min }=0.923, T_{\max }=0.991$

13200 measured reflections

6870 independent reflections

4304 reflections with $I>2 \sigma(I)$

$R_{\text {int }}=0.026$

$\theta_{\max }=32.8^{\circ}, \theta_{\min }=2.2^{\circ}$

$h=-12 \rightarrow 10$

$k=-13 \rightarrow 14$

$l=-20 \rightarrow 19$

Secondary atom site location: dual

Hydrogen site location: mixed

$\mathrm{H}$-atom parameters constrained

$w=1 /\left[\sigma^{2}\left(F_{\mathrm{o}}^{2}\right)+(0.0633 P)^{2}+0.1206 P\right]$

where $P=\left(F_{\mathrm{o}}{ }^{2}+2 F_{\mathrm{c}}{ }^{2}\right) / 3$

$(\Delta / \sigma)_{\max }<0.001$

$\Delta \rho_{\max }=0.34 \mathrm{e} \AA^{-3}$

$\Delta \rho_{\min }=-0.37$ e $\AA^{-3}$ 


\section{Special details}

Geometry. All esds (except the esd in the dihedral angle between two 1.s. planes) are estimated using the full covariance matrix. The cell esds are taken into account individually in the estimation of esds in distances, angles and torsion angles; correlations between esds in cell parameters are only used when they are defined by crystal symmetry. An approximate (isotropic) treatment of cell esds is used for estimating esds involving l.s. planes.

Fractional atomic coordinates and isotropic or equivalent isotropic displacement parameters $\left(\AA^{2}\right)$

\begin{tabular}{|c|c|c|c|c|}
\hline & $x$ & $y$ & $z$ & $U_{\text {iso }} * / U_{\text {eq }}$ \\
\hline $\mathrm{S} 1$ & $0.21006(5)$ & $0.11226(4)$ & $0.49970(3)$ & $0.04597(14)$ \\
\hline $\mathrm{O} 1$ & $0.20546(16)$ & $0.42592(12)$ & $0.58010(10)$ & $0.0542(3)$ \\
\hline $\mathrm{O} 2$ & $0.2688(3)$ & $-0.0438(2)$ & $1.00683(16)$ & $0.1150(8)$ \\
\hline $\mathrm{O} 3$ & $0.1551(3)$ & $0.16259(18)$ & $1.06193(12)$ & $0.0894(5)$ \\
\hline $\mathrm{O} 4$ & $0.21136(17)$ & $0.03480(13)$ & $0.42186(11)$ & 0.0618 (4) \\
\hline O5 & $0.25664(18)$ & $0.02943(13)$ & $0.59697(11)$ & $0.0624(4)$ \\
\hline N1 & $0.33461(16)$ & $0.22121(13)$ & $0.45549(10)$ & $0.0404(3)$ \\
\hline $\mathrm{N} 2$ & $0.3882(2)$ & $0.31933(16)$ & $0.69243(11)$ & 0.0543 (4) \\
\hline $\mathrm{C} 1$ & $0.4352(2)$ & $0.24222(16)$ & $0.52607(12)$ & 0.0407 (3) \\
\hline $\mathrm{H} 1$ & 0.484975 & 0.145601 & 0.564865 & $0.049^{*}$ \\
\hline $\mathrm{C} 2$ & $0.3309(2)$ & $0.33501(16)$ & $0.60307(13)$ & 0.0435 (4) \\
\hline $\mathrm{C} 3$ & $0.2942(3)$ & $0.4081(2)$ & $0.76676(14)$ & $0.0622(5)$ \\
\hline $\mathrm{H} 3 \mathrm{~A}$ & 0.212739 & 0.487753 & 0.733487 & $0.075^{*}$ \\
\hline H3B & 0.367076 & 0.450568 & 0.791143 & $0.075^{*}$ \\
\hline $\mathrm{C} 4$ & $0.2099(3)$ & $0.3155(2)$ & $0.85723(15)$ & $0.0598(5)$ \\
\hline $\mathrm{H} 4 \mathrm{~A}$ & 0.126979 & 0.283509 & 0.834039 & $0.072 *$ \\
\hline H4B & 0.155721 & 0.374364 & 0.908049 & $0.072 *$ \\
\hline $\mathrm{C} 5$ & $0.3330(3)$ & 0.1809 (2) & 0.90679 (14) & $0.0595(5)$ \\
\hline H5 & 0.409068 & 0.216659 & 0.934903 & $0.071^{*}$ \\
\hline C6 & $0.4334(3)$ & $0.0960(2)$ & $0.82547(15)$ & $0.0631(5)$ \\
\hline H6A & 0.517049 & 0.016237 & 0.856466 & $0.076^{*}$ \\
\hline H6B & 0.362540 & 0.054116 & 0.798864 & $0.076^{*}$ \\
\hline $\mathrm{C} 7$ & $0.5139(3)$ & $0.1963(2)$ & $0.73772(15)$ & $0.0617(5)$ \\
\hline $\mathrm{H} 7 \mathrm{~A}$ & 0.590425 & 0.233317 & 0.763271 & $0.074 *$ \\
\hline H7B & 0.574515 & 0.141684 & 0.685678 & $0.074 *$ \\
\hline $\mathrm{C} 8$ & $0.2494(3)$ & $0.0852(2)$ & $0.99538(17)$ & $0.0714(6)$ \\
\hline C9 & $0.0650(4)$ & 0.0879 (3) & $1.1529(2)$ & $0.1069(10)$ \\
\hline H9A & 0.004626 & 0.029494 & 1.132728 & $0.128^{*}$ \\
\hline H9B & 0.140898 & 0.023064 & 1.198758 & $0.128^{*}$ \\
\hline $\mathrm{C} 10$ & $-0.0456(5)$ & 0.1958 (4) & $1.2040(2)$ & $0.1357(14)$ \\
\hline H10A & 0.012297 & 0.263307 & 1.213528 & $0.204 *$ \\
\hline H10B & -0.091589 & 0.148539 & 1.269779 & $0.204^{*}$ \\
\hline $\mathrm{H} 10 \mathrm{C}$ & -0.131975 & 0.248210 & 1.163132 & $0.204 *$ \\
\hline C11 & $0.5719(2)$ & $0.31422(19)$ & $0.46630(14)$ & $0.0503(4)$ \\
\hline H11A & 0.636931 & 0.256036 & 0.418334 & $0.075^{*}$ \\
\hline H11B & 0.639730 & 0.321567 & 0.513158 & $0.075^{*}$ \\
\hline $\mathrm{H} 11 \mathrm{C}$ & 0.525388 & 0.410564 & 0.429625 & $0.075^{*}$ \\
\hline $\mathrm{C} 12$ & $0.0118(2)$ & $0.2217(2)$ & $0.5200(2)$ & $0.0700(6)$ \\
\hline $\mathrm{H} 12 \mathrm{~A}$ & -0.017746 & 0.283965 & 0.455966 & $0.105^{*}$ \\
\hline
\end{tabular}




$\begin{array}{lllll}\text { H12B } & 0.008836 & 0.280954 & 0.568219 & 0.105^{*} \\ \text { H12C } & -0.064229 & 0.159986 & 0.546811 & 0.105^{*} \\ \text { C13 } & 0.3206(2) & 0.31143(16) & 0.35381(13) & 0.0431(4) \\ \text { C14 } & 0.2231(2) & 0.45197(18) & 0.33966(14) & 0.0543(4) \\ \text { H14 } & 0.166814 & 0.491483 & 0.396039 & 0.065^{*} \\ \text { C15 } & 0.2097(3) & 0.5336(2) & 0.24101(16) & 0.0641(5) \\ \text { H15 } & 0.144679 & 0.628604 & 0.232086 & 0.077^{*} \\ \text { C16 } & 0.2899(3) & 0.4784(2) & 0.15543(15) & 0.0617(5) \\ \text { C17 } & 0.3898(3) & 0.3385(2) & 0.17173(16) & 0.0675(6) \\ \text { H17 } & 0.447572 & 0.299605 & 0.115395 & 0.081^{*} \\ \text { C18 } & 0.4060(3) & 0.25519(19) & 0.26953(14) & 0.0569(5) \\ \text { H18 } & 0.474265 & 0.161520 & 0.278607 & 0.068^{*} \\ \text { C19 } & 0.2674(4) & 0.5688(3) & 0.04839(17) & 0.0908(8) \\ \text { H19A } & 0.162225 & 0.569619 & 0.032294 & 0.136^{*} \\ \text { H19B } & 0.351973 & 0.526722 & -0.000565 & 0.136^{*} \\ \text { H19C } & 0.273737 & 0.667492 & 0.045582 & 0.136^{*}\end{array}$

Atomic displacement parameters $\left(\AA^{2}\right)$

\begin{tabular}{lllllll}
\hline & $U^{11}$ & $U^{22}$ & $U^{33}$ & $U^{12}$ & $U^{13}$ & $U^{23}$ \\
\hline S1 & $0.0381(2)$ & $0.0362(2)$ & $0.0636(3)$ & $-0.00995(16)$ & $-0.00197(19)$ & $-0.01207(17)$ \\
O1 & $0.0444(7)$ & $0.0491(6)$ & $0.0645(8)$ & $0.0048(5)$ & $-0.0079(6)$ & $-0.0194(5)$ \\
O2 & $0.157(2)$ & $0.0689(11)$ & $0.1072(15)$ & $-0.0226(12)$ & $0.0015(14)$ & $-0.0131(10)$ \\
O3 & $0.1050(15)$ & $0.0800(10)$ & $0.0695(10)$ & $-0.0174(10)$ & $0.0144(9)$ & $-0.0133(8)$ \\
O4 & $0.0598(9)$ & $0.0532(7)$ & $0.0829(9)$ & $-0.0212(6)$ & $-0.0043(7)$ & $-0.0280(6)$ \\
O5 & $0.0629(9)$ & $0.0520(7)$ & $0.0669(8)$ & $-0.0211(6)$ & $-0.0053(7)$ & $0.0039(6)$ \\
N1 & $0.0352(7)$ & $0.0367(6)$ & $0.0492(7)$ & $-0.0082(5)$ & $-0.0054(6)$ & $-0.0089(5)$ \\
N2 & $0.0541(10)$ & $0.0534(8)$ & $0.0517(8)$ & $0.0017(7)$ & $-0.0076(7)$ & $-0.0191(6)$ \\
C1 & $0.0333(8)$ & $0.0376(7)$ & $0.0507(9)$ & $-0.0047(6)$ & $-0.0051(7)$ & $-0.0119(6)$ \\
C2 & $0.0389(9)$ & $0.0382(7)$ & $0.0527(9)$ & $-0.0075(6)$ & $-0.0036(7)$ & $-0.0114(6)$ \\
C3 & $0.0761(15)$ & $0.0538(10)$ & $0.0557(11)$ & $-0.0053(10)$ & $-0.0049(10)$ & $-0.0226(8)$ \\
C4 & $0.0598(13)$ & $0.0600(11)$ & $0.0568(11)$ & $-0.0007(9)$ & $-0.0072(9)$ & $-0.0214(9)$ \\
C5 & $0.0600(13)$ & $0.0647(11)$ & $0.0542(11)$ & $-0.0060(9)$ & $-0.0145(9)$ & $-0.0170(8)$ \\
C6 & $0.0635(14)$ & $0.0587(10)$ & $0.0620(12)$ & $0.0094(9)$ & $-0.0241(10)$ & $-0.0167(9)$ \\
C7 & $0.0490(12)$ & $0.0749(12)$ & $0.0598(11)$ & $0.0038(9)$ & $-0.0152(9)$ & $-0.0247(9)$ \\
C8 & $0.0784(17)$ & $0.0663(13)$ & $0.0668(13)$ & $-0.0071(11)$ & $-0.0187(12)$ & $-0.0122(10)$ \\
C9 & $0.114(3)$ & $0.0948(18)$ & $0.0859(19)$ & $-0.0194(18)$ & $0.0136(18)$ & $0.0053(15)$ \\
C10 & $0.125(3)$ & $0.143(3)$ & $0.092(2)$ & $-0.013(2)$ & $0.033(2)$ & $0.009(2)$ \\
C11 & $0.0363(9)$ & $0.0539(9)$ & $0.0644(11)$ & $-0.0139(7)$ & $-0.0001(8)$ & $-0.0200(8)$ \\
C12 & $0.0332(10)$ & $0.0571(11)$ & $0.1202(19)$ & $-0.0110(8)$ & $0.0035(11)$ & $-0.0288(11)$ \\
C13 & $0.0396(9)$ & $0.0410(8)$ & $0.0493(9)$ & $-0.0094(7)$ & $-0.0059(7)$ & $-0.0105(6)$ \\
C14 & $0.0528(11)$ & $0.0484(9)$ & $0.0554(10)$ & $0.0016(8)$ & $-0.0105(9)$ & $-0.0109(8)$ \\
C15 & $0.0567(13)$ & $0.0568(11)$ & $0.0695(13)$ & $0.0007(9)$ & $-0.0188(10)$ & $-0.0030(9)$ \\
C16 & $0.0607(13)$ & $0.0713(12)$ & $0.0540(11)$ & $-0.0235(10)$ & $-0.0112(10)$ & $-0.0024(9)$ \\
C17 & $0.0791(16)$ & $0.0693(12)$ & $0.0534(11)$ & $-0.0223(11)$ & $0.0062(10)$ & $-0.0168(9)$ \\
C18 & $0.0627(13)$ & $0.0471(9)$ & $0.0573(11)$ & $-0.0109(8)$ & $0.0035(9)$ & $-0.0143(8)$ \\
C19 & $0.092(2)$ & $0.1060(19)$ & $0.0624(14)$ & $-0.0224(16)$ & $-0.0167(14)$ & $0.0085(13)$ \\
& & & & & & \\
\hline & & & & & &
\end{tabular}


Geometric parameters $\left(\AA,{ }^{\circ}\right)$

\begin{tabular}{|c|c|c|c|}
\hline $\mathrm{S} 1-\mathrm{O} 5$ & $1.4285(14)$ & $\mathrm{C} 7-\mathrm{H} 7 \mathrm{~A}$ & 0.9700 \\
\hline $\mathrm{S} 1-\mathrm{O} 4$ & $1.4286(13)$ & $\mathrm{C} 7-\mathrm{H} 7 \mathrm{~B}$ & 0.9700 \\
\hline $\mathrm{S} 1-\mathrm{N} 1$ & $1.6278(13)$ & $\mathrm{C} 9-\mathrm{C} 10$ & $1.428(4)$ \\
\hline $\mathrm{S} 1-\mathrm{C} 12$ & $1.7526(19)$ & C9-H9A & 0.9700 \\
\hline $\mathrm{O} 1-\mathrm{C} 2$ & $1.227(2)$ & C9-H9B & 0.9700 \\
\hline $\mathrm{O} 2-\mathrm{C} 8$ & $1.188(3)$ & $\mathrm{C} 10-\mathrm{H} 10 \mathrm{~A}$ & 0.9600 \\
\hline $\mathrm{O} 3-\mathrm{C} 8$ & $1.324(3)$ & $\mathrm{C} 10-\mathrm{H} 10 \mathrm{~B}$ & 0.9600 \\
\hline $\mathrm{O} 3-\mathrm{C} 9$ & $1.462(3)$ & $\mathrm{C} 10-\mathrm{H} 10 \mathrm{C}$ & 0.9600 \\
\hline $\mathrm{N} 1-\mathrm{C} 13$ & $1.445(2)$ & $\mathrm{C} 11-\mathrm{H} 11 \mathrm{~A}$ & 0.9600 \\
\hline $\mathrm{N} 1-\mathrm{C} 1$ & $1.476(2)$ & $\mathrm{C} 11-\mathrm{H} 11 \mathrm{~B}$ & 0.9600 \\
\hline $\mathrm{N} 2-\mathrm{C} 2$ & $1.346(2)$ & $\mathrm{C} 11-\mathrm{H} 11 \mathrm{C}$ & 0.9600 \\
\hline $\mathrm{N} 2-\mathrm{C} 3$ & $1.464(2)$ & $\mathrm{C} 12-\mathrm{H} 12 \mathrm{~A}$ & 0.9600 \\
\hline $\mathrm{N} 2-\mathrm{C} 7$ & $1.466(2)$ & $\mathrm{C} 12-\mathrm{H} 12 \mathrm{~B}$ & 0.9600 \\
\hline $\mathrm{C} 1-\mathrm{C} 11$ & $1.519(2)$ & $\mathrm{C} 12-\mathrm{H} 12 \mathrm{C}$ & 0.9600 \\
\hline $\mathrm{C} 1-\mathrm{C} 2$ & $1.537(2)$ & $\mathrm{C} 13-\mathrm{C} 14$ & $1.380(2)$ \\
\hline $\mathrm{C} 1-\mathrm{H} 1$ & 0.9800 & $\mathrm{C} 13-\mathrm{C} 18$ & $1.381(2)$ \\
\hline $\mathrm{C} 3-\mathrm{C} 4$ & $1.516(3)$ & $\mathrm{C} 14-\mathrm{C} 15$ & $1.382(3)$ \\
\hline $\mathrm{C} 3-\mathrm{H} 3 \mathrm{~A}$ & 0.9700 & C14-H14 & 0.9300 \\
\hline C $3-\mathrm{H} 3 \mathrm{~B}$ & 0.9700 & $\mathrm{C} 15-\mathrm{C} 16$ & $1.379(3)$ \\
\hline $\mathrm{C} 4-\mathrm{C} 5$ & $1.535(3)$ & C15-H15 & 0.9300 \\
\hline $\mathrm{C} 4-\mathrm{H} 4 \mathrm{~A}$ & 0.9700 & $\mathrm{C} 16-\mathrm{C} 17$ & $1.384(3)$ \\
\hline $\mathrm{C} 4-\mathrm{H} 4 \mathrm{~B}$ & 0.9700 & $\mathrm{C} 16-\mathrm{C} 19$ & $1.514(3)$ \\
\hline $\mathrm{C} 5-\mathrm{C} 8$ & $1.514(3)$ & $\mathrm{C} 17-\mathrm{C} 18$ & $1.381(3)$ \\
\hline $\mathrm{C} 5-\mathrm{C} 6$ & $1.526(3)$ & C17-H17 & 0.9300 \\
\hline $\mathrm{C} 5-\mathrm{H} 5$ & 0.9800 & C18-H18 & 0.9300 \\
\hline $\mathrm{C} 6-\mathrm{C} 7$ & $1.521(3)$ & C19-H19A & 0.9600 \\
\hline C6-H6A & 0.9700 & C19-H19B & 0.9600 \\
\hline $\mathrm{C} 6-\mathrm{H} 6 \mathrm{~B}$ & 0.9700 & $\mathrm{C} 19-\mathrm{H} 19 \mathrm{C}$ & 0.9600 \\
\hline $\mathrm{O} 5-\mathrm{S} 1-\mathrm{O} 4$ & $118.43(8)$ & $\mathrm{O} 2-\mathrm{C} 8-\mathrm{O} 3$ & $123.4(2)$ \\
\hline $\mathrm{O} 5-\mathrm{S} 1-\mathrm{N} 1$ & $106.30(8)$ & $\mathrm{O} 2-\mathrm{C} 8-\mathrm{C} 5$ & $125.1(2)$ \\
\hline $\mathrm{O} 4-\mathrm{S} 1-\mathrm{N} 1$ & $108.16(8)$ & $\mathrm{O} 3-\mathrm{C} 8-\mathrm{C} 5$ & $111.50(18)$ \\
\hline $\mathrm{O} 5-\mathrm{S} 1-\mathrm{C} 12$ & $108.50(11)$ & $\mathrm{C} 10-\mathrm{C} 9-\mathrm{O} 3$ & $108.7(2)$ \\
\hline $\mathrm{O} 4-\mathrm{S} 1-\mathrm{C} 12$ & $107.33(10)$ & $\mathrm{C} 10-\mathrm{C} 9-\mathrm{H} 9 \mathrm{~A}$ & 110.0 \\
\hline $\mathrm{N} 1-\mathrm{S} 1-\mathrm{C} 12$ & $107.71(8)$ & $\mathrm{O} 3-\mathrm{C} 9-\mathrm{H} 9 \mathrm{~A}$ & 110.0 \\
\hline $\mathrm{C} 8-\mathrm{O} 3-\mathrm{C} 9$ & $119.1(2)$ & $\mathrm{C} 10-\mathrm{C} 9-\mathrm{H} 9 \mathrm{~B}$ & 110.0 \\
\hline $\mathrm{C} 13-\mathrm{N} 1-\mathrm{C} 1$ & $122.65(12)$ & $\mathrm{O} 3-\mathrm{C} 9-\mathrm{H} 9 \mathrm{~B}$ & 110.0 \\
\hline $\mathrm{C} 13-\mathrm{N} 1-\mathrm{S} 1$ & $117.57(11)$ & $\mathrm{H} 9 \mathrm{~A}-\mathrm{C} 9-\mathrm{H} 9 \mathrm{~B}$ & 108.3 \\
\hline $\mathrm{C} 1-\mathrm{N} 1-\mathrm{S} 1$ & $118.92(10)$ & $\mathrm{C} 9-\mathrm{C} 10-\mathrm{H} 10 \mathrm{~A}$ & 109.5 \\
\hline $\mathrm{C} 2-\mathrm{N} 2-\mathrm{C} 3$ & $119.21(16)$ & $\mathrm{C} 9-\mathrm{C} 10-\mathrm{H} 10 \mathrm{~B}$ & 109.5 \\
\hline $\mathrm{C} 2-\mathrm{N} 2-\mathrm{C} 7$ & $126.42(15)$ & $\mathrm{H} 10 \mathrm{~A}-\mathrm{C} 10-\mathrm{H} 10 \mathrm{~B}$ & 109.5 \\
\hline $\mathrm{C} 3-\mathrm{N} 2-\mathrm{C} 7$ & $112.45(15)$ & $\mathrm{C} 9-\mathrm{C} 10-\mathrm{H} 10 \mathrm{C}$ & 109.5 \\
\hline $\mathrm{N} 1-\mathrm{C} 1-\mathrm{C} 11$ & $110.73(13)$ & $\mathrm{H} 10 \mathrm{~A}-\mathrm{C} 10-\mathrm{H} 10 \mathrm{C}$ & 109.5 \\
\hline $\mathrm{N} 1-\mathrm{C} 1-\mathrm{C} 2$ & $111.57(13)$ & $\mathrm{H} 10 \mathrm{~B}-\mathrm{C} 10-\mathrm{H} 10 \mathrm{C}$ & 109.5 \\
\hline $\mathrm{C} 11-\mathrm{C} 1-\mathrm{C} 2$ & $109.61(12)$ & $\mathrm{C} 1-\mathrm{C} 11-\mathrm{H} 11 \mathrm{~A}$ & 109.5 \\
\hline $\mathrm{N} 1-\mathrm{C} 1-\mathrm{H} 1$ & 108.3 & $\mathrm{C} 1-\mathrm{C} 11-\mathrm{H} 11 \mathrm{~B}$ & 109.5 \\
\hline
\end{tabular}




\begin{tabular}{|c|c|c|c|}
\hline $\mathrm{C} 11-\mathrm{C} 1-\mathrm{H} 1$ & 108.3 & $\mathrm{H} 11 \mathrm{~A}-\mathrm{C} 11-\mathrm{H} 11 \mathrm{~B}$ & 109.5 \\
\hline $\mathrm{C} 2-\mathrm{C} 1-\mathrm{H} 1$ & 108.3 & $\mathrm{C} 1-\mathrm{C} 11-\mathrm{H} 11 \mathrm{C}$ & 109.5 \\
\hline $\mathrm{O} 1-\mathrm{C} 2-\mathrm{N} 2$ & $121.94(15)$ & $\mathrm{H} 11 \mathrm{~A}-\mathrm{C} 11-\mathrm{H} 11 \mathrm{C}$ & 109.5 \\
\hline $\mathrm{O} 1-\mathrm{C} 2-\mathrm{C} 1$ & $120.33(15)$ & $\mathrm{H} 11 \mathrm{~B}-\mathrm{C} 11-\mathrm{H} 11 \mathrm{C}$ & 109.5 \\
\hline $\mathrm{N} 2-\mathrm{C} 2-\mathrm{C} 1$ & $117.58(15)$ & $\mathrm{S} 1-\mathrm{C} 12-\mathrm{H} 12 \mathrm{~A}$ & 109.5 \\
\hline $\mathrm{N} 2-\mathrm{C} 3-\mathrm{C} 4$ & $110.73(15)$ & $\mathrm{S} 1-\mathrm{C} 12-\mathrm{H} 12 \mathrm{~B}$ & 109.5 \\
\hline $\mathrm{N} 2-\mathrm{C} 3-\mathrm{H} 3 \mathrm{~A}$ & 109.5 & $\mathrm{H} 12 \mathrm{~A}-\mathrm{C} 12-\mathrm{H} 12 \mathrm{~B}$ & 109.5 \\
\hline $\mathrm{C} 4-\mathrm{C} 3-\mathrm{H} 3 \mathrm{~A}$ & 109.5 & $\mathrm{~S} 1-\mathrm{C} 12-\mathrm{H} 12 \mathrm{C}$ & 109.5 \\
\hline $\mathrm{N} 2-\mathrm{C} 3-\mathrm{H} 3 \mathrm{~B}$ & 109.5 & $\mathrm{H} 12 \mathrm{~A}-\mathrm{C} 12-\mathrm{H} 12 \mathrm{C}$ & 109.5 \\
\hline $\mathrm{C} 4-\mathrm{C} 3-\mathrm{H} 3 \mathrm{~B}$ & 109.5 & $\mathrm{H} 12 \mathrm{~B}-\mathrm{C} 12-\mathrm{H} 12 \mathrm{C}$ & 109.5 \\
\hline $\mathrm{H} 3 \mathrm{~A}-\mathrm{C} 3-\mathrm{H} 3 \mathrm{~B}$ & 108.1 & $\mathrm{C} 14-\mathrm{C} 13-\mathrm{C} 18$ & $119.67(16)$ \\
\hline $\mathrm{C} 3-\mathrm{C} 4-\mathrm{C} 5$ & $111.24(18)$ & $\mathrm{C} 14-\mathrm{C} 13-\mathrm{N} 1$ & $121.20(15)$ \\
\hline $\mathrm{C} 3-\mathrm{C} 4-\mathrm{H} 4 \mathrm{~A}$ & 109.4 & $\mathrm{C} 18-\mathrm{C} 13-\mathrm{N} 1$ & $119.13(14)$ \\
\hline $\mathrm{C} 5-\mathrm{C} 4-\mathrm{H} 4 \mathrm{~A}$ & 109.4 & $\mathrm{C} 13-\mathrm{C} 14-\mathrm{C} 15$ & $119.47(17)$ \\
\hline $\mathrm{C} 3-\mathrm{C} 4-\mathrm{H} 4 \mathrm{~B}$ & 109.4 & $\mathrm{C} 13-\mathrm{C} 14-\mathrm{H} 14$ & 120.3 \\
\hline $\mathrm{C} 5-\mathrm{C} 4-\mathrm{H} 4 \mathrm{~B}$ & 109.4 & $\mathrm{C} 15-\mathrm{C} 14-\mathrm{H} 14$ & 120.3 \\
\hline $\mathrm{H} 4 \mathrm{~A}-\mathrm{C} 4-\mathrm{H} 4 \mathrm{~B}$ & 108.0 & $\mathrm{C} 16-\mathrm{C} 15-\mathrm{C} 14$ & $122.01(18)$ \\
\hline $\mathrm{C} 8-\mathrm{C} 5-\mathrm{C} 6$ & $112.44(17)$ & $\mathrm{C} 16-\mathrm{C} 15-\mathrm{H} 15$ & 119.0 \\
\hline $\mathrm{C} 8-\mathrm{C} 5-\mathrm{C} 4$ & $111.67(19)$ & $\mathrm{C} 14-\mathrm{C} 15-\mathrm{H} 15$ & 119.0 \\
\hline $\mathrm{C} 6-\mathrm{C} 5-\mathrm{C} 4$ & $110.22(16)$ & $\mathrm{C} 15-\mathrm{C} 16-\mathrm{C} 17$ & $117.39(18)$ \\
\hline $\mathrm{C} 8-\mathrm{C} 5-\mathrm{H} 5$ & 107.4 & $\mathrm{C} 15-\mathrm{C} 16-\mathrm{C} 19$ & $120.6(2)$ \\
\hline $\mathrm{C} 6-\mathrm{C} 5-\mathrm{H} 5$ & 107.4 & $\mathrm{C} 17-\mathrm{C} 16-\mathrm{C} 19$ & $122.0(2)$ \\
\hline $\mathrm{C} 4-\mathrm{C} 5-\mathrm{H} 5$ & 107.4 & $\mathrm{C} 18-\mathrm{C} 17-\mathrm{C} 16$ & $121.65(19)$ \\
\hline $\mathrm{C} 7-\mathrm{C} 6-\mathrm{C} 5$ & $110.66(16)$ & $\mathrm{C} 18-\mathrm{C} 17-\mathrm{H} 17$ & 119.2 \\
\hline $\mathrm{C} 7-\mathrm{C} 6-\mathrm{H} 6 \mathrm{~A}$ & 109.5 & $\mathrm{C} 16-\mathrm{C} 17-\mathrm{H} 17$ & 119.2 \\
\hline $\mathrm{C} 5-\mathrm{C} 6-\mathrm{H} 6 \mathrm{~A}$ & 109.5 & $\mathrm{C} 13-\mathrm{C} 18-\mathrm{C} 17$ & $119.76(17)$ \\
\hline $\mathrm{C} 7-\mathrm{C} 6-\mathrm{H} 6 \mathrm{~B}$ & 109.5 & $\mathrm{C} 13-\mathrm{C} 18-\mathrm{H} 18$ & 120.1 \\
\hline $\mathrm{C} 5-\mathrm{C} 6-\mathrm{H} 6 \mathrm{~B}$ & 109.5 & $\mathrm{C} 17-\mathrm{C} 18-\mathrm{H} 18$ & 120.1 \\
\hline $\mathrm{H} 6 \mathrm{~A}-\mathrm{C} 6-\mathrm{H} 6 \mathrm{~B}$ & 108.1 & $\mathrm{C} 16-\mathrm{C} 19-\mathrm{H} 19 \mathrm{~A}$ & 109.5 \\
\hline $\mathrm{N} 2-\mathrm{C} 7-\mathrm{C} 6$ & $109.70(17)$ & C16-C19-H19B & 109.5 \\
\hline $\mathrm{N} 2-\mathrm{C} 7-\mathrm{H} 7 \mathrm{~A}$ & 109.7 & $\mathrm{H} 19 \mathrm{~A}-\mathrm{C} 19-\mathrm{H} 19 \mathrm{~B}$ & 109.5 \\
\hline $\mathrm{C} 6-\mathrm{C} 7-\mathrm{H} 7 \mathrm{~A}$ & 109.7 & $\mathrm{C} 16-\mathrm{C} 19-\mathrm{H} 19 \mathrm{C}$ & 109.5 \\
\hline $\mathrm{N} 2-\mathrm{C} 7-\mathrm{H} 7 \mathrm{~B}$ & 109.7 & $\mathrm{H} 19 \mathrm{~A}-\mathrm{C} 19-\mathrm{H} 19 \mathrm{C}$ & 109.5 \\
\hline $\mathrm{C} 6-\mathrm{C} 7-\mathrm{H} 7 \mathrm{~B}$ & 109.7 & $\mathrm{H} 19 \mathrm{~B}-\mathrm{C} 19-\mathrm{H} 19 \mathrm{C}$ & 109.5 \\
\hline $\mathrm{H} 7 \mathrm{~A}-\mathrm{C} 7-\mathrm{H} 7 \mathrm{~B}$ & 108.2 & & \\
\hline $\mathrm{O} 5-\mathrm{S} 1-\mathrm{N} 1-\mathrm{C} 13$ & $176.09(11)$ & $\mathrm{C} 2-\mathrm{N} 2-\mathrm{C} 7-\mathrm{C} 6$ & $-103.4(2)$ \\
\hline $\mathrm{O} 4-\mathrm{S} 1-\mathrm{N} 1-\mathrm{C} 13$ & $47.92(13)$ & $\mathrm{C} 3-\mathrm{N} 2-\mathrm{C} 7-\mathrm{C} 6$ & $60.5(2)$ \\
\hline $\mathrm{C} 12-\mathrm{S} 1-\mathrm{N} 1-\mathrm{C} 13$ & $-67.78(14)$ & $\mathrm{C} 5-\mathrm{C} 6-\mathrm{C} 7-\mathrm{N} 2$ & $-57.7(2)$ \\
\hline $\mathrm{O} 5-\mathrm{S} 1-\mathrm{N} 1-\mathrm{C} 1$ & $-14.24(14)$ & $\mathrm{C} 9-\mathrm{O} 3-\mathrm{C} 8-\mathrm{O} 2$ & $-2.2(4)$ \\
\hline $\mathrm{O} 4-\mathrm{S} 1-\mathrm{N} 1-\mathrm{C} 1$ & $-142.40(12)$ & $\mathrm{C} 9-\mathrm{O} 3-\mathrm{C} 8-\mathrm{C} 5$ & $-179.8(2)$ \\
\hline $\mathrm{C} 12-\mathrm{S} 1-\mathrm{N} 1-\mathrm{C} 1$ & $101.89(14)$ & $\mathrm{C} 6-\mathrm{C} 5-\mathrm{C} 8-\mathrm{O} 2$ & $6.3(4)$ \\
\hline $\mathrm{C} 13-\mathrm{N} 1-\mathrm{C} 1-\mathrm{C} 11$ & $-25.36(19)$ & $\mathrm{C} 4-\mathrm{C} 5-\mathrm{C} 8-\mathrm{O} 2$ & $130.8(3)$ \\
\hline $\mathrm{S} 1-\mathrm{N} 1-\mathrm{C} 1-\mathrm{C} 11$ & $165.51(11)$ & $\mathrm{C} 6-\mathrm{C} 5-\mathrm{C} 8-\mathrm{O} 3$ & $-176.2(2)$ \\
\hline $\mathrm{C} 13-\mathrm{N} 1-\mathrm{C} 1-\mathrm{C} 2$ & $97.02(16)$ & $\mathrm{C} 4-\mathrm{C} 5-\mathrm{C} 8-\mathrm{O} 3$ & $-51.7(3)$ \\
\hline $\mathrm{S} 1-\mathrm{N} 1-\mathrm{C} 1-\mathrm{C} 2$ & $-72.11(15)$ & $\mathrm{C} 8-\mathrm{O} 3-\mathrm{C} 9-\mathrm{C} 10$ & $-171.3(3)$ \\
\hline $\mathrm{C} 3-\mathrm{N} 2-\mathrm{C} 2-\mathrm{O} 1$ & $3.0(3)$ & $\mathrm{C} 1-\mathrm{N} 1-\mathrm{C} 13-\mathrm{C} 14$ & $-76.6(2)$ \\
\hline $\mathrm{C} 7-\mathrm{N} 2-\mathrm{C} 2-\mathrm{O} 1$ & $165.98(18)$ & $\mathrm{S} 1-\mathrm{N} 1-\mathrm{C} 13-\mathrm{C} 14$ & $92.68(17)$ \\
\hline
\end{tabular}




$\begin{array}{llll}\mathrm{C} 3-\mathrm{N} 2-\mathrm{C} 2-\mathrm{C} 1 & 178.66(15) & \mathrm{C} 1-\mathrm{N} 1-\mathrm{C} 13-\mathrm{C} 18 & 103.66(19) \\ \mathrm{C} 7-\mathrm{N} 2-\mathrm{C} 2-\mathrm{C} 1 & -18.4(3) & \mathrm{S} 1-\mathrm{N} 1-\mathrm{C} 13-\mathrm{C} 18 & -87.07(18) \\ \mathrm{N} 1-\mathrm{C} 1-\mathrm{C} 2-\mathrm{O} 1 & -28.1(2) & \mathrm{C} 18-\mathrm{C} 13-\mathrm{C} 14-\mathrm{C} 15 & 1.3(3) \\ \mathrm{C} 11-\mathrm{C} 1-\mathrm{C} 2-\mathrm{O} 1 & 94.89(19) & \mathrm{N} 1-\mathrm{C} 13-\mathrm{C} 14-\mathrm{C} 15 & -178.45(17) \\ \mathrm{N} 1-\mathrm{C} 1-\mathrm{C} 2-\mathrm{N} 2 & 156.17(14) & \mathrm{C} 13-\mathrm{C} 14-\mathrm{C} 15-\mathrm{C} 16 & 0.7(3) \\ \mathrm{C} 11-\mathrm{C} 1-\mathrm{C} 2-\mathrm{N} 2 & -80.81(18) & \mathrm{C} 14-\mathrm{C} 15-\mathrm{C} 16-\mathrm{C} 17 & -2.1(3) \\ \mathrm{C} 2-\mathrm{N} 2-\mathrm{C} 3-\mathrm{C} 4 & 106.1(2) & \mathrm{C} 14-\mathrm{C} 15-\mathrm{C} 16-\mathrm{C} 19 & 177.7(2) \\ \mathrm{C} 7-\mathrm{N} 2-\mathrm{C} 3-\mathrm{C} 4 & -59.1(2) & \mathrm{C} 15-\mathrm{C} 16-\mathrm{C} 17-\mathrm{C} 18 & 1.7(3) \\ \mathrm{N} 2-\mathrm{C} 3-\mathrm{C} 4-\mathrm{C} 5 & 54.5(2) & \mathrm{C} 19-\mathrm{C} 16-\mathrm{C} 17-\mathrm{C} 18 & -178.1(2) \\ \mathrm{C} 3-\mathrm{C} 4-\mathrm{C} 5-\mathrm{C} 8 & -178.38(17) & \mathrm{C} 14-\mathrm{C} 13-\mathrm{C} 18-\mathrm{C} 17 & -1.7(3) \\ \mathrm{C} 3-\mathrm{C} 4-\mathrm{C} 5-\mathrm{C} 6 & -52.6(2) & \mathrm{N} 1-\mathrm{C} 13-\mathrm{C} 18-\mathrm{C} 17 & 178.02(17) \\ \mathrm{C} 8-\mathrm{C} 5-\mathrm{C} 6-\mathrm{C} 7 & 179.54(18) & \mathrm{C} 16-\mathrm{C} 17-\mathrm{C} 18-\mathrm{C} 13 & 0.2(3) \\ \mathrm{C} 4-\mathrm{C} 5-\mathrm{C} 6-\mathrm{C} 7 & 54.2(2) & & \end{array}$

Hydrogen-bond geometry $\left(A,{ }^{\circ}\right)$

\begin{tabular}{lllll}
\hline$D-\mathrm{H} \cdots A$ & $D-\mathrm{H}$ & $\mathrm{H} \cdots A$ & $D \cdots A$ & $D-\mathrm{H} \cdots A$ \\
\hline $\mathrm{C} 12-\mathrm{H} 12 B \cdots \mathrm{O} 1$ & 0.96 & 2.50 & $3.210(2)$ & 130 \\
$\mathrm{C} 12-\mathrm{H} 12 C \cdots \mathrm{O} 4{ }^{\mathrm{i}}$ & 0.96 & 2.44 & $3.376(2)$ & 164 \\
$\mathrm{C} 14-\mathrm{H} 14 \cdots \mathrm{O} 1$ & 0.93 & 2.48 & $3.177(2)$ & 132 \\
\hline
\end{tabular}

Symmetry code: (i) $-x,-y,-z+1$.

1-[N-(4-methylphenyl)-N-(methylsulfonyl)alanyl]piperidine-4-carboxylic acid (II)

Crystal data

$\mathrm{C}_{17} \mathrm{H}_{24} \mathrm{~N}_{2} \mathrm{O}_{5} \mathrm{~S}$

$M_{r}=368.44$

Monoclinic, $P 2_{1} / n$

$a=12.1013(2) \AA$

$b=12.3092(2) \AA$

$c=12.4348(3) \AA$

$\beta=100.546(2)^{\circ}$

$V=1820.97(6) \AA^{3}$

$Z=4$

\section{Data collection}

Oxford Diffraction Xcalibur, Gemini Ultra R diffractometer

Radiation source: fine-focus sealed X-ray tube Graphite monochromator

Detector resolution: 10.3712 pixels $\mathrm{mm}^{-1}$

$\omega$ scans

Absorption correction: analytical

[CrysAlisPro (Rigaku OD, 2018), based on expressions derived by Clark \& Reid (1995)]

\section{Refinement}

Refinement on $F^{2}$

Least-squares matrix: full

$R\left[F^{2}>2 \sigma\left(F^{2}\right)\right]=0.043$

$w R\left(F^{2}\right)=0.126$

$S=1.02$
$F(000)=784$

$D_{\mathrm{x}}=1.344 \mathrm{Mg} \mathrm{m}^{-3}$

Mo $K \alpha$ radiation, $\lambda=0.71073 \AA$

Cell parameters from 8359 reflections

$\theta=2.7-31.5^{\circ}$

$\mu=0.21 \mathrm{~mm}^{-1}$

$T=295 \mathrm{~K}$

Plate, colorless

$0.77 \times 0.18 \times 0.11 \mathrm{~mm}$

$T_{\min }=0.882, T_{\max }=0.980$

29518 measured reflections

6284 independent reflections

4779 reflections with $I>2 \sigma(I)$

$R_{\text {int }}=0.026$

$\theta_{\text {max }}=32.7^{\circ}, \theta_{\text {min }}=2.2^{\circ}$

$h=-18 \rightarrow 18$

$k=-18 \rightarrow 17$

$l=-17 \rightarrow 18$

6284 reflections

232 parameters

0 restraints

Primary atom site location: dual

Secondary atom site location: dual 
Hydrogen site location: difference Fourier map $\mathrm{H}$ atoms treated by a mixture of independent and constrained refinement

$$
\begin{aligned}
& w=1 /\left[\sigma^{2}\left(F_{\mathrm{o}}^{2}\right)+(0.0593 P)^{2}+0.3685 P\right] \\
& \text { where } P=\left(F_{\mathrm{o}}^{2}+2 F_{\mathrm{c}}^{2}\right) / 3 \\
& (\Delta / \sigma)_{\max }=0.001 \\
& \Delta \rho_{\max }=0.29 \mathrm{e} \AA^{-3} \\
& \Delta \rho_{\min }=-0.29 \mathrm{e}^{-3}
\end{aligned}
$$

Special details

Geometry. All esds (except the esd in the dihedral angle between two 1.s. planes) are estimated using the full covariance

\begin{tabular}{|c|c|c|c|c|}
\hline & $x$ & $y$ & $z$ & $U_{\text {iso }} * / U_{\text {eq }}$ \\
\hline $\mathrm{S} 1$ & $0.58667(2)$ & $0.35673(2)$ & $0.60942(2)$ & $0.03513(9)$ \\
\hline $\mathrm{O} 1$ & $0.68078(9)$ & $0.19851(8)$ & $0.41698(10)$ & $0.0531(3)$ \\
\hline $\mathrm{O} 2$ & $1.04956(12)$ & $0.60661(11)$ & $0.37887(13)$ & 0.0780 \\
\hline $\mathrm{O} 3$ & $1.13713(10)$ & $0.46783(11)$ & $0.31848(13)$ & $0.0732(4)$ \\
\hline H3 & $1.195(2)$ & $0.509(2)$ & $0.352(2)$ & $0.110^{*}$ \\
\hline $\mathrm{O} 4$ & $0.50845(8)$ & $0.37442(9)$ & $0.68112(8)$ & $0.0479(2)$ \\
\hline $\mathrm{O} 5$ & $0.65804(8)$ & $0.44541(8)$ & $0.59029(9)$ & $0.0501(2)$ \\
\hline N1 & $0.51748(8)$ & $0.32221(9)$ & $0.48974(8)$ & $0.0344(2)$ \\
\hline N2 & $0.73982(9)$ & $0.33804(10)$ & $0.32395(10)$ & $0.0447(3)$ \\
\hline $\mathrm{C} 1$ & $0.56084(10)$ & $0.35539(10)$ & $0.39054(10)$ & $0.0359(2)$ \\
\hline H1 & 0.580679 & 0.432597 & 0.397240 & $0.043 *$ \\
\hline $\mathrm{C} 17$ & $0.67424(14)$ & $0.24889(13)$ & $0.66359(13)$ & $0.0555(4)$ \\
\hline H17A & 0.726630 & 0.233560 & 0.616194 & $0.083 *$ \\
\hline H17B & 0.629402 & 0.185582 & 0.669483 & $0.083 *$ \\
\hline $\mathrm{H} 17 \mathrm{C}$ & 0.714621 & 0.268508 & 0.734742 & $0.083 *$ \\
\hline $\mathrm{C} 2$ & $0.66661(10)$ & $0.29079(11)$ & $0.37903(10)$ & 0.0392 \\
\hline $\mathrm{C} 3$ & 0.83897 (11) & $0.27729(13)$ & $0.30503(13)$ & 0.0488 \\
\hline $\mathrm{H} 3 \mathrm{~A}$ & 0.832698 & 0.263103 & 0.227419 & $0.059 *$ \\
\hline H3B & 0.842307 & 0.208002 & 0.342640 & $0.059 *$ \\
\hline $\mathrm{C} 4$ & $0.94599(11)$ & $0.34095(12)$ & $0.34608(11)$ & $0.0427(3)$ \\
\hline $\mathrm{H} 4 \mathrm{~A}$ & 1.009763 & 0.302094 & 0.327725 & $0.051 *$ \\
\hline H4B & 0.957054 & 0.347251 & 0.425085 & $0.051^{*}$ \\
\hline $\mathrm{C} 5$ & $0.94003(11)$ & $0.45455(12)$ & $0.29524(11)$ & 0.0438 \\
\hline H5 & 0.933185 & 0.446018 & 0.215935 & $0.053 *$ \\
\hline C6 & $0.83536(12)$ & $0.51356(12)$ & $0.31663(13)$ & 0.0510 \\
\hline H6A & 0.841595 & 0.526101 & 0.394520 & $0.061 *$ \\
\hline H6B & 0.829392 & 0.583548 & 0.280182 & $0.061 *$ \\
\hline $\mathrm{C} 7$ & $0.73079(12)$ & $0.44690(14)$ & $0.27497(12)$ & 0.0510 \\
\hline H7A & 0.665534 & 0.483641 & 0.292748 & $0.061 *$ \\
\hline H7B & 0.720557 & 0.440587 & 0.196007 & $0.061 *$ \\
\hline $\mathrm{C} 8$ & 1.04549 (13) & $0.51874(13)$ & $0.33621(12)$ & 0.0489 \\
\hline C9 & $0.47176(12)$ & $0.34023(14)$ & $0.28791(12)$ & 0.0489 \\
\hline H9A & 0.458064 & 0.264097 & 0.275037 & $0.073^{*}$ \\
\hline H9B & 0.497692 & 0.371751 & 0.226417 & $0.073 *$ \\
\hline
\end{tabular}
matrix. The cell esds are taken into account individually in the estimation of esds in distances, angles and torsion angles; correlations between esds in cell parameters are only used when they are defined by crystal symmetry. An approximate (isotropic) treatment of cell esds is used for estimating esds involving l.s. planes.

Fractional atomic coordinates and isotropic or equivalent isotropic displacement parameters $\left(\AA^{2}\right)$ 


$\begin{array}{lllll}\text { H9C } & 0.403409 & 0.375286 & 0.297628 & 0.073^{*} \\ \text { C10 } & 0.42232(10) & 0.25039(10) & 0.48451(10) & 0.0364(2) \\ \text { C11 } & 0.43049(14) & 0.14088(12) & 0.46161(15) & 0.0541(4) \\ \text { H11 } & 0.498708 & 0.111490 & 0.451688 & 0.065^{*} \\ \text { C12 } & 0.33581(16) & 0.07526(14) & 0.45357(15) & 0.0641(4) \\ \text { H12 } & 0.341460 & 0.001934 & 0.437384 & 0.077^{*} \\ \text { C13 } & 0.23387(13) & 0.11605(14) & 0.46894(12) & 0.0553(4) \\ \text { C14 } & 0.22767(12) & 0.22528(14) & 0.49295(13) & 0.0519(4) \\ \text { H14 } & 0.159834 & 0.254119 & 0.504670 & 0.062^{*} \\ \text { C15 } & 0.32057(10) & 0.29283(12) & 0.49995(11) & 0.0423(3) \\ \text { H15 } & 0.314406 & 0.366372 & 0.514958 & 0.051^{*} \\ \text { C16 } & 0.13157(17) & 0.0435(2) & 0.45871(17) & 0.0843(7) \\ \text { H16A } & 0.085792 & 0.066468 & 0.509939 & 0.126^{*} \\ \text { H16B } & 0.155060 & -0.030246 & 0.473939 & 0.126^{*} \\ \text { H16C } & 0.088916 & 0.048332 & 0.385744 & 0.126^{*}\end{array}$

Atomic displacement parameters $\left(\AA^{2}\right)$

\begin{tabular}{|c|c|c|c|c|c|c|}
\hline & $U^{11}$ & $U^{22}$ & $U^{33}$ & $U^{12}$ & $U^{13}$ & $U^{23}$ \\
\hline S1 & $0.03062(14)$ & $0.03481(15)$ & $0.03976(16)$ & $-0.00400(11)$ & $0.00588(11)$ & $-0.00067(11)$ \\
\hline $\mathrm{O} 1$ & $0.0514(6)$ & $0.0410(5)$ & $0.0722(7)$ & $0.0074(4)$ & $0.0248(5)$ & $0.0058(5)$ \\
\hline $\mathrm{O} 2$ & $0.0771(9)$ & $0.0640(8)$ & $0.0943(10)$ & $-0.0098(7)$ & $0.0193(7)$ & $-0.0239(7)$ \\
\hline $\mathrm{O} 3$ & $0.0402(6)$ & $0.0697(8)$ & $0.1097(11)$ & $-0.0137(5)$ & $0.0139(6)$ & $-0.0303(7)$ \\
\hline $\mathrm{O} 4$ & $0.0448(5)$ & $0.0571(6)$ & $0.0436(5)$ & $-0.0033(4)$ & $0.0129(4)$ & $-0.0075(4)$ \\
\hline O5 & $0.0436(5)$ & $0.0481(5)$ & $0.0563(6)$ & $-0.0190(4)$ & $0.0033(4)$ & $0.0010(4)$ \\
\hline N1 & 0.0293 (4) & $0.0375(5)$ & $0.0373(5)$ & $-0.0058(4)$ & $0.0082(4)$ & $-0.0016(4)$ \\
\hline $\mathrm{N} 2$ & $0.0353(5)$ & $0.0490(6)$ & $0.0530(6)$ & $0.0044(5)$ & $0.0171(5)$ & $0.0037(5)$ \\
\hline $\mathrm{C} 1$ & $0.0321(5)$ & $0.0382(6)$ & $0.0387(6)$ & $-0.0001(5)$ & $0.0102(4)$ & $0.0020(5)$ \\
\hline $\mathrm{C} 17$ & $0.0548(8)$ & $0.0583(9)$ & $0.0515(8)$ & $0.0155(7)$ & $0.0051(7)$ & $0.0093(7)$ \\
\hline $\mathrm{C} 2$ & $0.0345(6)$ & $0.0428(6)$ & $0.0414(6)$ & $0.0005(5)$ & $0.0103(5)$ & $-0.0025(5)$ \\
\hline $\mathrm{C} 3$ & $0.0366(6)$ & $0.0525(8)$ & $0.0609(9)$ & $0.0005(6)$ & $0.0184(6)$ & $-0.0099(7)$ \\
\hline $\mathrm{C} 4$ & $0.0360(6)$ & $0.0486(7)$ & $0.0450(7)$ & $0.0029(5)$ & $0.0114(5)$ & $-0.0049(5)$ \\
\hline $\mathrm{C} 5$ & $0.0388(6)$ & $0.0551(8)$ & $0.0387(6)$ & $-0.0029(6)$ & $0.0107(5)$ & $0.0008(6)$ \\
\hline C6 & $0.0493(8)$ & $0.0481(8)$ & $0.0591(8)$ & $0.0065(6)$ & $0.0190(7)$ & $0.0149(6)$ \\
\hline $\mathrm{C} 7$ & $0.0397(7)$ & $0.0658(9)$ & $0.0499(8)$ & $0.0079(6)$ & $0.0147(6)$ & $0.0195(7)$ \\
\hline $\mathrm{C} 8$ & $0.0490(8)$ & $0.0517(8)$ & $0.0469(7)$ & $-0.0062(6)$ & $0.0107(6)$ & $-0.0010(6)$ \\
\hline C9 & 0.0407 (7) & $0.0635(9)$ & $0.0411(7)$ & $0.0017(6)$ & $0.0039(5)$ & $0.0014(6)$ \\
\hline $\mathrm{C} 10$ & $0.0327(5)$ & $0.0359(6)$ & $0.0404(6)$ & $-0.0076(5)$ & $0.0059(5)$ & $0.0002(5)$ \\
\hline C11 & $0.0499(8)$ & $0.0397(7)$ & $0.0735(10)$ & $-0.0066(6)$ & $0.0131(7)$ & $-0.0083(7)$ \\
\hline $\mathrm{C} 12$ & $0.0713(11)$ & $0.0434(8)$ & $0.0759(11)$ & $-0.0228(8)$ & $0.0088(9)$ & $-0.0078(7)$ \\
\hline $\mathrm{C} 13$ & $0.0513(8)$ & $0.0660(9)$ & $0.0443(7)$ & $-0.0296(7)$ & $-0.0026(6)$ & $0.0081(7)$ \\
\hline $\mathrm{C} 14$ & $0.0316(6)$ & $0.0692(10)$ & $0.0534(8)$ & $-0.0112(6)$ & $0.0038(5)$ & $0.0085(7)$ \\
\hline $\mathrm{C} 15$ & $0.0320(6)$ & $0.0447(7)$ & $0.0500(7)$ & $-0.0045(5)$ & $0.0066(5)$ & $0.0028(5)$ \\
\hline $\mathrm{C} 16$ & $0.0732(12)$ & $0.1026(16)$ & $0.0695(11)$ & $-0.0573(12)$ & $-0.0065(9)$ & $0.0100(11)$ \\
\hline
\end{tabular}


Geometric parameters $\left(\AA,{ }^{\circ}\right)$

\begin{tabular}{|c|c|c|c|}
\hline $\mathrm{S} 1-\mathrm{O} 4$ & $1.4309(10)$ & $\mathrm{C} 5-\mathrm{C} 8$ & $1.508(2)$ \\
\hline $\mathrm{S} 1-\mathrm{O} 5$ & $1.4385(10)$ & $\mathrm{C} 5-\mathrm{C} 6$ & $1.5252(19)$ \\
\hline $\mathrm{S} 1-\mathrm{N} 1$ & $1.6249(10)$ & $\mathrm{C} 5-\mathrm{H} 5$ & 0.9800 \\
\hline $\mathrm{S} 1-\mathrm{C} 17$ & $1.7543(15)$ & $\mathrm{C} 6-\mathrm{C} 7$ & $1.517(2)$ \\
\hline $\mathrm{O} 1-\mathrm{C} 2$ & $1.2300(16)$ & $\mathrm{C} 6-\mathrm{H} 6 \mathrm{~A}$ & 0.9700 \\
\hline $\mathrm{O} 2-\mathrm{C} 8$ & $1.2018(19)$ & C6-H6B & 0.9700 \\
\hline $\mathrm{O} 3-\mathrm{C} 8$ & $1.3269(19)$ & $\mathrm{C} 7-\mathrm{H} 7 \mathrm{~A}$ & 0.9700 \\
\hline $\mathrm{O} 3-\mathrm{H} 3$ & $0.90(3)$ & $\mathrm{C} 7-\mathrm{H} 7 \mathrm{~B}$ & 0.9700 \\
\hline $\mathrm{N} 1-\mathrm{C} 10$ & $1.4438(15)$ & C9-H9A & 0.9600 \\
\hline $\mathrm{N} 1-\mathrm{C} 1$ & $1.4834(15)$ & C9-H9B & 0.9600 \\
\hline $\mathrm{N} 2-\mathrm{C} 2$ & $1.3470(17)$ & $\mathrm{C} 9-\mathrm{H} 9 \mathrm{C}$ & 0.9600 \\
\hline $\mathrm{N} 2-\mathrm{C} 7$ & 1.4677 (19) & $\mathrm{C} 10-\mathrm{C} 15$ & $1.3829(18)$ \\
\hline $\mathrm{N} 2-\mathrm{C} 3$ & $1.4688(17)$ & $\mathrm{C} 10-\mathrm{C} 11$ & $1.3850(19)$ \\
\hline $\mathrm{C} 1-\mathrm{C} 9$ & $1.5238(18)$ & $\mathrm{C} 11-\mathrm{C} 12$ & $1.390(2)$ \\
\hline $\mathrm{C} 1-\mathrm{C} 2$ & $1.5356(17)$ & $\mathrm{C} 11-\mathrm{H} 11$ & 0.9300 \\
\hline $\mathrm{C} 1-\mathrm{H} 1$ & 0.9800 & $\mathrm{C} 12-\mathrm{C} 13$ & $1.377(3)$ \\
\hline C17-H17A & 0.9600 & $\mathrm{C} 12-\mathrm{H} 12$ & 0.9300 \\
\hline C17-H17B & 0.9600 & $\mathrm{C} 13-\mathrm{C} 14$ & $1.382(2)$ \\
\hline $\mathrm{C} 17-\mathrm{H} 17 \mathrm{C}$ & 0.9600 & $\mathrm{C} 13-\mathrm{C} 16$ & $1.513(2)$ \\
\hline $\mathrm{C} 3-\mathrm{C} 4$ & $1.5198(19)$ & $\mathrm{C} 14-\mathrm{C} 15$ & $1.3882(18)$ \\
\hline $\mathrm{C} 3-\mathrm{H} 3 \mathrm{~A}$ & 0.9700 & $\mathrm{C} 14-\mathrm{H} 14$ & 0.9300 \\
\hline $\mathrm{C} 3-\mathrm{H} 3 \mathrm{~B}$ & 0.9700 & C15-H15 & 0.9300 \\
\hline $\mathrm{C} 4-\mathrm{C} 5$ & $1.531(2)$ & $\mathrm{C} 16-\mathrm{H} 16 \mathrm{~A}$ & 0.9600 \\
\hline $\mathrm{C} 4-\mathrm{H} 4 \mathrm{~A}$ & 0.9700 & C16-H16B & 0.9600 \\
\hline $\mathrm{C} 4-\mathrm{H} 4 \mathrm{~B}$ & 0.9700 & $\mathrm{C} 16-\mathrm{H} 16 \mathrm{C}$ & 0.9600 \\
\hline $\mathrm{O} 4-\mathrm{S} 1-\mathrm{O} 5$ & $118.24(6)$ & $\mathrm{C} 7-\mathrm{C} 6-\mathrm{C} 5$ & $110.50(13)$ \\
\hline $\mathrm{O} 4-\mathrm{S} 1-\mathrm{N} 1$ & $108.76(6)$ & $\mathrm{C} 7-\mathrm{C} 6-\mathrm{H} 6 \mathrm{~A}$ & 109.5 \\
\hline $\mathrm{O} 5-\mathrm{S} 1-\mathrm{N} 1$ & $105.74(6)$ & $\mathrm{C} 5-\mathrm{C} 6-\mathrm{H} 6 \mathrm{~A}$ & 109.5 \\
\hline $\mathrm{O} 4-\mathrm{S} 1-\mathrm{C} 17$ & $107.33(7)$ & $\mathrm{C} 7-\mathrm{C} 6-\mathrm{H} 6 \mathrm{~B}$ & 109.5 \\
\hline $\mathrm{O} 5-\mathrm{S} 1-\mathrm{C} 17$ & $107.40(7)$ & $\mathrm{C} 5-\mathrm{C} 6-\mathrm{H} 6 \mathrm{~B}$ & 109.5 \\
\hline $\mathrm{N} 1-\mathrm{S} 1-\mathrm{C} 17$ & $109.13(7)$ & $\mathrm{H} 6 \mathrm{~A}-\mathrm{C} 6-\mathrm{H} 6 \mathrm{~B}$ & 108.1 \\
\hline $\mathrm{C} 8-\mathrm{O} 3-\mathrm{H} 3$ & $105.0(16)$ & $\mathrm{N} 2-\mathrm{C} 7-\mathrm{C} 6$ & $110.97(12)$ \\
\hline $\mathrm{C} 10-\mathrm{N} 1-\mathrm{C} 1$ & $122.13(10)$ & $\mathrm{N} 2-\mathrm{C} 7-\mathrm{H} 7 \mathrm{~A}$ & 109.4 \\
\hline $\mathrm{C} 10-\mathrm{N} 1-\mathrm{S} 1$ & $118.32(8)$ & $\mathrm{C} 6-\mathrm{C} 7-\mathrm{H} 7 \mathrm{~A}$ & 109.4 \\
\hline $\mathrm{C} 1-\mathrm{N} 1-\mathrm{S} 1$ & $119.22(8)$ & $\mathrm{N} 2-\mathrm{C} 7-\mathrm{H} 7 \mathrm{~B}$ & 109.4 \\
\hline $\mathrm{C} 2-\mathrm{N} 2-\mathrm{C} 7$ & $126.82(11)$ & $\mathrm{C} 6-\mathrm{C} 7-\mathrm{H} 7 \mathrm{~B}$ & 109.4 \\
\hline $\mathrm{C} 2-\mathrm{N} 2-\mathrm{C} 3$ & $119.62(12)$ & $\mathrm{H} 7 \mathrm{~A}-\mathrm{C} 7-\mathrm{H} 7 \mathrm{~B}$ & 108.0 \\
\hline $\mathrm{C} 7-\mathrm{N} 2-\mathrm{C} 3$ & $113.53(11)$ & $\mathrm{O} 2-\mathrm{C} 8-\mathrm{O} 3$ & $122.04(15)$ \\
\hline $\mathrm{N} 1-\mathrm{C} 1-\mathrm{C} 9$ & $111.04(10)$ & $\mathrm{O} 2-\mathrm{C} 8-\mathrm{C} 5$ & $125.76(15)$ \\
\hline $\mathrm{N} 1-\mathrm{C} 1-\mathrm{C} 2$ & $111.26(10)$ & $\mathrm{O} 3-\mathrm{C} 8-\mathrm{C} 5$ & $112.20(13)$ \\
\hline $\mathrm{C} 9-\mathrm{C} 1-\mathrm{C} 2$ & $109.42(11)$ & $\mathrm{C} 1-\mathrm{C} 9-\mathrm{H} 9 \mathrm{~A}$ & 109.5 \\
\hline $\mathrm{N} 1-\mathrm{C} 1-\mathrm{H} 1$ & 108.3 & $\mathrm{C} 1-\mathrm{C} 9-\mathrm{H} 9 \mathrm{~B}$ & 109.5 \\
\hline $\mathrm{C} 9-\mathrm{C} 1-\mathrm{H} 1$ & 108.3 & $\mathrm{H} 9 \mathrm{~A}-\mathrm{C} 9-\mathrm{H} 9 \mathrm{~B}$ & 109.5 \\
\hline $\mathrm{C} 2-\mathrm{C} 1-\mathrm{H} 1$ & 108.3 & $\mathrm{C} 1-\mathrm{C} 9-\mathrm{H} 9 \mathrm{C}$ & 109.5 \\
\hline $\mathrm{S} 1-\mathrm{C} 17-\mathrm{H} 17 \mathrm{~A}$ & 109.5 & $\mathrm{H} 9 \mathrm{~A}-\mathrm{C} 9-\mathrm{H} 9 \mathrm{C}$ & 109.5 \\
\hline
\end{tabular}




\begin{tabular}{|c|c|c|c|}
\hline $\mathrm{S} 1-\mathrm{C} 17-\mathrm{H} 17 \mathrm{~B}$ & 109.5 & $\mathrm{H} 9 \mathrm{~B}-\mathrm{C} 9-\mathrm{H} 9 \mathrm{C}$ & 109.5 \\
\hline $\mathrm{H} 17 \mathrm{~A}-\mathrm{C} 17-\mathrm{H} 17 \mathrm{~B}$ & 109.5 & $\mathrm{C} 15-\mathrm{C} 10-\mathrm{C} 11$ & $119.68(12)$ \\
\hline $\mathrm{S} 1-\mathrm{C} 17-\mathrm{H} 17 \mathrm{C}$ & 109.5 & $\mathrm{C} 15-\mathrm{C} 10-\mathrm{N} 1$ & $119.04(11)$ \\
\hline $\mathrm{H} 17 \mathrm{~A}-\mathrm{C} 17-\mathrm{H} 17 \mathrm{C}$ & 109.5 & $\mathrm{C} 11-\mathrm{C} 10-\mathrm{N} 1$ & $121.26(12)$ \\
\hline $\mathrm{H} 17 \mathrm{~B}-\mathrm{C} 17-\mathrm{H} 17 \mathrm{C}$ & 109.5 & $\mathrm{C} 10-\mathrm{C} 11-\mathrm{C} 12$ & $119.43(15)$ \\
\hline $\mathrm{O} 1-\mathrm{C} 2-\mathrm{N} 2$ & $122.37(12)$ & $\mathrm{C} 10-\mathrm{C} 11-\mathrm{H} 11$ & 120.3 \\
\hline $\mathrm{O} 1-\mathrm{C} 2-\mathrm{C} 1$ & $120.17(11)$ & $\mathrm{C} 12-\mathrm{C} 11-\mathrm{H} 11$ & 120.3 \\
\hline $\mathrm{N} 2-\mathrm{C} 2-\mathrm{C} 1$ & $117.44(11)$ & $\mathrm{C} 13-\mathrm{C} 12-\mathrm{C} 11$ & $121.70(16)$ \\
\hline $\mathrm{N} 2-\mathrm{C} 3-\mathrm{C} 4$ & $110.77(12)$ & $\mathrm{C} 13-\mathrm{C} 12-\mathrm{H} 12$ & 119.1 \\
\hline $\mathrm{N} 2-\mathrm{C} 3-\mathrm{H} 3 \mathrm{~A}$ & 109.5 & $\mathrm{C} 11-\mathrm{C} 12-\mathrm{H} 12$ & 119.1 \\
\hline $\mathrm{C} 4-\mathrm{C} 3-\mathrm{H} 3 \mathrm{~A}$ & 109.5 & $\mathrm{C} 12-\mathrm{C} 13-\mathrm{C} 14$ & $118.04(13)$ \\
\hline $\mathrm{N} 2-\mathrm{C} 3-\mathrm{H} 3 \mathrm{~B}$ & 109.5 & $\mathrm{C} 12-\mathrm{C} 13-\mathrm{C} 16$ & $120.88(18)$ \\
\hline $\mathrm{C} 4-\mathrm{C} 3-\mathrm{H} 3 \mathrm{~B}$ & 109.5 & $\mathrm{C} 14-\mathrm{C} 13-\mathrm{C} 16$ & $121.08(18)$ \\
\hline $\mathrm{H} 3 \mathrm{~A}-\mathrm{C} 3-\mathrm{H} 3 \mathrm{~B}$ & 108.1 & $\mathrm{C} 13-\mathrm{C} 14-\mathrm{C} 15$ & $121.38(15)$ \\
\hline $\mathrm{C} 3-\mathrm{C} 4-\mathrm{C} 5$ & $111.06(12)$ & $\mathrm{C} 13-\mathrm{C} 14-\mathrm{H} 14$ & 119.3 \\
\hline $\mathrm{C} 3-\mathrm{C} 4-\mathrm{H} 4 \mathrm{~A}$ & 109.4 & $\mathrm{C} 15-\mathrm{C} 14-\mathrm{H} 14$ & 119.3 \\
\hline $\mathrm{C} 5-\mathrm{C} 4-\mathrm{H} 4 \mathrm{~A}$ & 109.4 & $\mathrm{C} 10-\mathrm{C} 15-\mathrm{C} 14$ & $119.76(14)$ \\
\hline $\mathrm{C} 3-\mathrm{C} 4-\mathrm{H} 4 \mathrm{~B}$ & 109.4 & $\mathrm{C} 10-\mathrm{C} 15-\mathrm{H} 15$ & 120.1 \\
\hline $\mathrm{C} 5-\mathrm{C} 4-\mathrm{H} 4 \mathrm{~B}$ & 109.4 & $\mathrm{C} 14-\mathrm{C} 15-\mathrm{H} 15$ & 120.1 \\
\hline $\mathrm{H} 4 \mathrm{~A}-\mathrm{C} 4-\mathrm{H} 4 \mathrm{~B}$ & 108.0 & $\mathrm{C} 13-\mathrm{C} 16-\mathrm{H} 16 \mathrm{~A}$ & 109.5 \\
\hline $\mathrm{C} 8-\mathrm{C} 5-\mathrm{C} 6$ & $111.72(13)$ & $\mathrm{C} 13-\mathrm{C} 16-\mathrm{H} 16 \mathrm{~B}$ & 109.5 \\
\hline $\mathrm{C} 8-\mathrm{C} 5-\mathrm{C} 4$ & $111.47(12)$ & $\mathrm{H} 16 \mathrm{~A}-\mathrm{C} 16-\mathrm{H} 16 \mathrm{~B}$ & 109.5 \\
\hline $\mathrm{C} 6-\mathrm{C} 5-\mathrm{C} 4$ & $109.92(11)$ & $\mathrm{C} 13-\mathrm{C} 16-\mathrm{H} 16 \mathrm{C}$ & 109.5 \\
\hline $\mathrm{C} 8-\mathrm{C} 5-\mathrm{H} 5$ & 107.9 & $\mathrm{H} 16 \mathrm{~A}-\mathrm{C} 16-\mathrm{H} 16 \mathrm{C}$ & 109.5 \\
\hline $\mathrm{C} 6-\mathrm{C} 5-\mathrm{H} 5$ & 107.9 & $\mathrm{H} 16 \mathrm{~B}-\mathrm{C} 16-\mathrm{H} 16 \mathrm{C}$ & 109.5 \\
\hline $\mathrm{C} 4-\mathrm{C} 5-\mathrm{H} 5$ & 107.9 & & \\
\hline $\mathrm{O} 4-\mathrm{S} 1-\mathrm{N} 1-\mathrm{C} 10$ & $-39.35(11)$ & $\mathrm{C} 8-\mathrm{C} 5-\mathrm{C} 6-\mathrm{C} 7$ & $-179.78(12)$ \\
\hline $\mathrm{O} 5-\mathrm{S} 1-\mathrm{N} 1-\mathrm{C} 10$ & $-167.31(9)$ & $\mathrm{C} 4-\mathrm{C} 5-\mathrm{C} 6-\mathrm{C} 7$ & $-55.49(15)$ \\
\hline $\mathrm{C} 17-\mathrm{S} 1-\mathrm{N} 1-\mathrm{C} 10$ & $77.45(11)$ & $\mathrm{C} 2-\mathrm{N} 2-\mathrm{C} 7-\mathrm{C} 6$ & $125.15(15)$ \\
\hline $\mathrm{O} 4-\mathrm{S} 1-\mathrm{N} 1-\mathrm{C} 1$ & $147.15(9)$ & $\mathrm{C} 3-\mathrm{N} 2-\mathrm{C} 7-\mathrm{C} 6$ & $-56.90(16)$ \\
\hline $\mathrm{O} 5-\mathrm{S} 1-\mathrm{N} 1-\mathrm{C} 1$ & $19.20(11)$ & $\mathrm{C} 5-\mathrm{C} 6-\mathrm{C} 7-\mathrm{N} 2$ & $56.02(16)$ \\
\hline $\mathrm{C} 17-\mathrm{S} 1-\mathrm{N} 1-\mathrm{C} 1$ & $-96.05(11)$ & $\mathrm{C} 6-\mathrm{C} 5-\mathrm{C} 8-\mathrm{O} 2$ & $-1.4(2)$ \\
\hline $\mathrm{C} 10-\mathrm{N} 1-\mathrm{C} 1-\mathrm{C} 9$ & $20.64(16)$ & $\mathrm{C} 4-\mathrm{C} 5-\mathrm{C} 8-\mathrm{O} 2$ & $-124.79(17)$ \\
\hline $\mathrm{S} 1-\mathrm{N} 1-\mathrm{C} 1-\mathrm{C} 9$ & $-166.13(9)$ & $\mathrm{C} 6-\mathrm{C} 5-\mathrm{C} 8-\mathrm{O} 3$ & $179.68(13)$ \\
\hline $\mathrm{C} 10-\mathrm{N} 1-\mathrm{C} 1-\mathrm{C} 2$ & $-101.50(13)$ & $\mathrm{C} 4-\mathrm{C} 5-\mathrm{C} 8-\mathrm{O} 3$ & $56.26(17)$ \\
\hline $\mathrm{S} 1-\mathrm{N} 1-\mathrm{C} 1-\mathrm{C} 2$ & $71.73(12)$ & $\mathrm{C} 1-\mathrm{N} 1-\mathrm{C} 10-\mathrm{C} 15$ & $-106.70(14)$ \\
\hline $\mathrm{C} 7-\mathrm{N} 2-\mathrm{C} 2-\mathrm{O} 1$ & $179.61(14)$ & $\mathrm{S} 1-\mathrm{N} 1-\mathrm{C} 10-\mathrm{C} 15$ & $80.01(14)$ \\
\hline $\mathrm{C} 3-\mathrm{N} 2-\mathrm{C} 2-\mathrm{O} 1$ & $1.8(2)$ & $\mathrm{C} 1-\mathrm{N} 1-\mathrm{C} 10-\mathrm{C} 11$ & $71.61(17)$ \\
\hline $\mathrm{C} 7-\mathrm{N} 2-\mathrm{C} 2-\mathrm{C} 1$ & $1.4(2)$ & $\mathrm{S} 1-\mathrm{N} 1-\mathrm{C} 10-\mathrm{C} 11$ & $-101.69(14)$ \\
\hline $\mathrm{C} 3-\mathrm{N} 2-\mathrm{C} 2-\mathrm{C} 1$ & $-176.43(12)$ & $\mathrm{C} 15-\mathrm{C} 10-\mathrm{C} 11-\mathrm{C} 12$ & $0.5(2)$ \\
\hline $\mathrm{N} 1-\mathrm{C} 1-\mathrm{C} 2-\mathrm{O} 1$ & $28.24(17)$ & $\mathrm{N} 1-\mathrm{C} 10-\mathrm{C} 11-\mathrm{C} 12$ & $-177.77(14)$ \\
\hline $\mathrm{C} 9-\mathrm{C} 1-\mathrm{C} 2-\mathrm{O} 1$ & $-94.83(15)$ & $\mathrm{C} 10-\mathrm{C} 11-\mathrm{C} 12-\mathrm{C} 13$ & $-0.7(3)$ \\
\hline $\mathrm{N} 1-\mathrm{C} 1-\mathrm{C} 2-\mathrm{N} 2$ & $-153.51(11)$ & $\mathrm{C} 11-\mathrm{C} 12-\mathrm{C} 13-\mathrm{C} 14$ & $0.0(3)$ \\
\hline $\mathrm{C} 9-\mathrm{C} 1-\mathrm{C} 2-\mathrm{N} 2$ & $83.42(15)$ & $\mathrm{C} 11-\mathrm{C} 12-\mathrm{C} 13-\mathrm{C} 16$ & $179.38(17)$ \\
\hline $\mathrm{C} 2-\mathrm{N} 2-\mathrm{C} 3-\mathrm{C} 4$ & $-125.84(14)$ & $\mathrm{C} 12-\mathrm{C} 13-\mathrm{C} 14-\mathrm{C} 15$ & $0.9(2)$ \\
\hline $\mathrm{C} 7-\mathrm{N} 2-\mathrm{C} 3-\mathrm{C} 4$ & $56.05(17)$ & $\mathrm{C} 16-\mathrm{C} 13-\mathrm{C} 14-\mathrm{C} 15$ & $-178.47(15)$ \\
\hline $\mathrm{N} 2-\mathrm{C} 3-\mathrm{C} 4-\mathrm{C} 5$ & $-54.68(15)$ & $\mathrm{C} 11-\mathrm{C} 10-\mathrm{C} 15-\mathrm{C} 14$ & $0.4(2)$ \\
\hline
\end{tabular}


supporting information

\begin{tabular}{llll}
$\mathrm{C} 3-\mathrm{C} 4-\mathrm{C} 5-\mathrm{C} 8$ & $179.52(11)$ & $\mathrm{N} 1-\mathrm{C} 10-\mathrm{C} 15-\mathrm{C} 14$ & $178.68(12)$ \\
$\mathrm{C} 3-\mathrm{C} 4-\mathrm{C} 5-\mathrm{C} 6$ & $55.07(15)$ & $\mathrm{C} 13-\mathrm{C} 14-\mathrm{C} 15-\mathrm{C} 10$ & $-1.1(2)$ \\
\hline
\end{tabular}

Hydrogen-bond geometry $\left(A,{ }^{\circ}\right)$

\begin{tabular}{lllll}
\hline$D-\mathrm{H} \cdots A$ & $D-\mathrm{H}$ & $\mathrm{H} \cdots A$ & $D \cdots A$ & $D-\mathrm{H} \cdots A$ \\
\hline $\mathrm{O} 3-\mathrm{H} 3 \cdots \mathrm{O} 5^{\mathrm{i}}$ & $0.90(3)$ & $1.88(3)$ & $2.7463(15)$ & $161(2)$ \\
$\mathrm{C} 17-\mathrm{H} 17 A \cdots \mathrm{O} 1$ & 0.96 & 2.48 & $3.144(2)$ & 127 \\
$\mathrm{C} 4-\mathrm{H} 4 B \cdots \mathrm{O} 2^{\mathrm{i}}$ & 0.97 & 2.52 & $3.471(2)$ & 167 \\
$\mathrm{C} 11-\mathrm{H} 11 \cdots \mathrm{O} 1$ & 0.93 & 2.56 & $3.2558(19)$ & 132
\end{tabular}

Symmetry code: (i) $-x+2,-y+1,-z+1$. 